\title{
FUSION SUBCATEGORIES OF REPRESENTATION CATEGORIES OF TWISTED QUANTUM DOUBLES OF FINITE GROUPS
}

\author{
DEEPAK NAIDU, DMITRI NIKSHYCH, AND SARAH WITHERSPOON
}

\begin{abstract}
We describe all fusion subcategories of the representation category $\operatorname{Rep}\left(D^{\omega}(G)\right)$ of a twisted quantum double $D^{\omega}(G)$, where $G$ is a finite group and $\omega$ is a 3-cocycle on $G$. In view of the fact that every group-theoretical braided fusion category can be embedded into some $\operatorname{Rep}\left(D^{\omega}(G)\right)$, this gives a complete description of all group-theoretical braided fusion categories. We describe the lattice and give formulas for some invariants of the fusion subcategories of $\operatorname{Rep}\left(D^{\omega}(G)\right)$. We also give a characterization of group-theoretical braided fusion categories as equivariantizations of pointed categories.
\end{abstract}

\section{INTRODUCTION}

Let $G$ be a finite group and $\omega$ be a 3-cocycle on $G$. In [DPR1, DPR2] Dijkgraaf, Pasquier, and Roche introduced a quasi-triangular quasi-Hopf algebra $D^{\omega}(G)$. When $\omega=1$ this quasiHopf algebra coincides with the Drinfeld double $D(G)$ of $G$ and so $D^{\omega}(G)$ is often called a twisted quantum double of $G$. It is well known that the representation category $\operatorname{Rep}\left(D^{\omega}(G)\right)$ of $D^{\omega}(G)$ is a modular category $[\mathrm{BK}]$ and is braided equivalent to the center $\mathcal{Z}\left(\mathrm{Vec}_{G}^{\omega}\right)$ of the tensor category $\operatorname{Vec}_{G}^{\omega}$ of finite-dimensional $G$-graded vector spaces with associativity constraint defined using $\omega[\mathrm{Mj}]$.

The principal goal of this paper is to give a complete description of fusion subcategories of $\operatorname{Rep}\left(D^{\omega}(G)\right)$ and the lattice formed by them. Our description of the lattice may shed more light on the structure of $\operatorname{Rep}\left(D^{\omega}(G)\right)$. For instance, the group of braided autoequivalences of $\operatorname{Rep}\left(D^{\omega}(G)\right)$ acts on this lattice, and so one can, in principle, derive some information about this group from our description.

Our results also have consequences more generally for a group-theoretical braided fusion category, that is a braided category $\mathcal{C}$ dual to $\operatorname{Vec}_{G}^{\omega}$ for some $G$ and $\omega$ (see Section 2.1 or [02]). Since $\mathcal{C}$ is braided, it can be realized as a fusion subcategory of $\operatorname{Rep}\left(D^{\omega}(G)\right) \cong \mathcal{Z}\left(\operatorname{Vec}_{G}^{\omega}\right)$. This means that our description of all fusion subcategories of $\operatorname{Rep}\left(D^{\omega}(G)\right)$, for all finite groups $G$ and 3-cocycles $\omega$, is equivalent to a description of all group-theoretical braided fusion categories. In particular, our results give a description of all modular [BK] group-theoretical fusion categories.

Group-theoretical fusion categories are of interest as examples of fusion categories that can be described explicitly in terms of finite groups and their cohomology [01]. They are also more general than one might at first expect: All semisimple quasi-Hopf algebras of prime power dimension have group-theoretical representation categories, a consequence of a more general result on nilpotent fusion categories [DGNO]. For some time it was unknown whether there are any complex semisimple Hopf algebras having non-group-theoretical representation categories; the first known example was announced recently by the second author $[\mathrm{Nk}$.

Date: November 11, 2018. 
1.1. Main results. Let $G$ be a finite group, and $k$ an algebraically closed field of characteristic zero. Unless otherwise stated all cocycles appearing in this work will have coefficients in the trivial module $k^{\times}$. All categories are assumed to be $k$-linear and semisimple with finite-dimensional Hom-spaces and finitely many isomorphism classes of simple objects. All functors are assumed to be additive and $k$-linear.

Theorem 1.1. Fusion subcategories of the representation category of the Drinfeld double $D(G)$ are in bijection with triples $(K, H, B)$, where $K, H$ are normal subgroups of $G$ centralizing each other and $B: K \times H \rightarrow k^{\times}$is a G-invariant bicharacter.

Theorem 1.1 gives a simple description of all fusion subcategories for the untwisted double $D(G)$. Now let $\omega \in Z^{3}\left(G, k^{\times}\right)$be a 3 -cocycle on $G$. In the twisted $(\omega \neq 1)$ case the notion of a $G$-invariant bicharacter must be twisted (see Definition 5.4).

Theorem 1.2. Fusion subcategories of the representation category of the twisted double $D^{\omega}(G)$ are in bijection with triples $(K, H, B)$ where $K, H$ are normal subgroups of $G$ centralizing each other and $B: K \times H \rightarrow k^{\times}$is a G-invariant $\omega$-bicharacter.

As noted earlier, the above result is equivalent to a description of all group-theoretical braided fusion categories.

Let $(K, H, B)$ be a triple as described in the above theorem and let $\mathcal{S}(K, H, B)$ denote the corresponding fusion subcategory of $\operatorname{Rep}\left(D^{\omega}(G)\right)$. The next result gives a criterion for the fusion subcategory $\mathcal{S}(K, H, B) \subseteq \operatorname{Rep}\left(D^{\omega}(G)\right)$ to be nondegenerate or prime. Recall [M1] that a nondegenerate braided fusion category is called prime if it has no proper nontrivial nondegenerate subcategories.

Theorem 1.3. $\quad$ (i) The fusion subcategory $\mathcal{S}(K, H, B) \subseteq \operatorname{Rep}\left(D^{\omega}(G)\right)$ is nondegenerate if and only if $H K=G$ and the symmetric bicharacter $\left.B B^{\mathrm{op}}\right|_{(K \cap H) \times(K \cap H)}$ is nondegenerate.

(ii) $\operatorname{Rep}\left(D^{\omega}(G)\right)$ is prime if and only if there is no triple $(K, H, B)$, where $K$ and $H$ are proper normal subgroups of $G$ that centralize each other, $H K=G$, and $B$ is a $G$-invariant $\omega$-bicharacter on $K \times H$ such that $\left.B B^{\mathrm{op}}\right|_{(K \cap H) \times(K \cap H)}$ is nondegenerate.

We note that, in our situation, being nondegenerate is equivalent to being modular (see Section (2.3). Therefore, the above result combined with Theorem 1.2 gives a description of all modular group-theoretical fusion categories.

1.2. Organization of the paper. Section 2 contains necessary preliminary information about fusion categories and module categories. We also recall definitions and results from [M1, DGNO] concerning centralizers in braided fusion categories.

Section 3 (respectively, Section 5) is devoted to a description of fusion subcategories of the representation category of the Drinfeld double (respectively, twisted double) of a finite group. We chose to treat the untwisted and twisted cases separately because the twisted case involves rather technical cohomological computations which may obscure the main results. We note that when $\omega=1$ the results of Section 5 reduce to those of Section 3.

Sections 3, 5, and 6 contain proofs of some of our main results stated above. Theorems 1.1. 1.2 and Theorem 1.3 correspond to Theorems 3.12, 5.11, and Theorem 6.7, respectively.

Section 4 (respectively, Section 6) is devoted to a description of the lattice and some invariants of fusion subcategories of the representation category of the Drinfeld double (respectively, twisted double) of a finite group. 
In Section 7 we prove that a braided fusion category is group-theoretical if and only if it is equivalent to an equivariantization of a pointed fusion category with respect to an action of a group $G$.

1.3. Acknowledgments. The second author was partially supported by NSA grant H9823007-1-0081 and NSF grant DMS-0800545. He is grateful to Vladimir Drinfeld, Pavel Etingof, and Viktor Ostrik for helpful discussions. The third author was partially supported by NSA grant H98230-07-1-0038 and NSF grant DMS-0800832.

\section{PRELIMinaRies}

2.1. Group-theoretical fusion categories. A fusion category over $k$ is a $k$-linear semisimple rigid tensor category with finitely many isomorphism classes of simple objects and finite dimensional Hom-spaces such that the neutral object is simple [ENO].

A fusion category is said to be pointed if all its simple objects are invertible. A typical example of a pointed category is $\operatorname{Vec}_{G}^{\omega}$, the category of finite dimensional vector spaces over $k$ graded by the finite group $G$. The morphisms in this category are linear transformations that respect the grading and the associativity constraint is given by the normalized 3-cocycle $\omega$ on $G$.

Consider the fusion category $\operatorname{Vec}_{G}^{\omega}$. Let $H$ be a subgroup of $G$ such that $\left.\omega\right|_{H \times H \times H}$ is cohomologically trivial. Let $\psi$ be a 2-cochain in $C^{2}\left(H, k^{\times}\right)$satisfying $\left.\omega\right|_{H \times H \times H}=\delta^{2} \psi$. The twisted group algebra $k^{\psi}[H]$ is an associative unital algebra in $\operatorname{Vec}_{G}^{\omega}$. Define $\mathcal{C}=$ $\mathcal{C}(G, \omega, H, \psi)$ to be the category of $k^{\psi}[H]$-bimodules in $\operatorname{Vec}_{G}^{\omega}$. Then $\mathcal{C}$ is a fusion category with tensor product $\otimes_{k^{\psi}[H]}$ and unit object $k^{\psi}[H]$. Categories of the form $\mathcal{C}(G, \omega, H, \psi)$ are known as group-theoretical [ENO, Defn. 8.40], [O2]. It is known that a fusion category $\mathcal{C}$ is group-theoretical if and only if it is dual to a pointed category with respect to an indecomposable module category. (See [O1] and [M3] for notions of module category and duality.)

2.2. Adjoint categories and central series of fusion categories. The following definitions were introduced in [GN, ENO].

Let $\mathcal{C}$ be a fusion category. The adjoint category $\mathcal{C}_{a d}$ of $\mathcal{C}$ is the full fusion subcategory of $\mathcal{C}$ generated by all subobjects of $X \otimes X^{*}$, where $X$ runs through simple objects of $\mathcal{C}$. For example, let $G$ be a finite group and let $\mathcal{C}=\operatorname{Rep}(G)$ be the representation category of $G$. Then $\mathcal{C}_{a d}=\operatorname{Rep}(G / Z(G))$, where $Z(G)$ is the center of $G$.

Let $\mathcal{C}^{(0)}=\mathcal{C}, \mathcal{C}^{(1)}=\mathcal{C}_{a d}$, and $\mathcal{C}^{(n)}=\left(\mathcal{C}^{(n-1)}\right)_{a d}$ for every integer $n \geq 1$.

The non-increasing sequence of fusion subcategories of $\mathcal{C}$

$$
\mathcal{C}=\mathcal{C}^{(0)} \supseteq \mathcal{C}^{(1)} \supseteq \cdots \supseteq \mathcal{C}^{(n)} \supseteq \cdots
$$

is called the upper central series of $\mathcal{C}$. This definition generalizes the classical one, as we show next: Let $G$ be a finite group with identity element $e$, and $\mathcal{C}=\operatorname{Rep}(G)$. Let

$$
\{e\}=C^{0}(G) \subseteq C^{1}(G) \subseteq \cdots \subseteq C^{n}(G) \subseteq \cdots
$$

be the upper central series of $G$; i.e., $C^{0}(G):=\{e\}, C^{1}(G):=Z(G)$ (the center of $G$ ) and for $n \geq 1$ the subgroup $C^{n}(G)$ is defined by $C^{n}(G) / C^{n-1}(G)=Z\left(G / C^{n-1}(G)\right)$. Then $\mathcal{C}^{(n)}=\operatorname{Rep}\left(G / C^{n}(G)\right)$.

Let $\mathcal{C}$ be a fusion category such that its Grothendieck ring $K_{0}(\mathcal{C})$ is commutative (e.g., $\mathcal{C}$ is braided). Let $\mathcal{D}$ be a fusion subcategory of $\mathcal{C}$. The commutator $\mathcal{D}^{c o}$ of $\mathcal{D}$ is defined to be 
the fusion subcategory of $\mathcal{C}$ generated by all simple objects $Y$ of $\mathcal{C}$ such that $Y \otimes Y^{*} \in \mathcal{D}$. An example: Let $G$ be a finite group and let $\mathcal{C}=\operatorname{Rep}(G)$. Any fusion subcategory of $\mathcal{C}$ is of the form $\operatorname{Rep}(G / N)$ for some normal subgroup $N$ of $G$. The simple objects of the category $\operatorname{Rep}(G / N)^{c o}$ are irreducible representations $Y$ of $G$ for which $Y \otimes Y^{*}$ restricts to the trivial representation of $N$.

Let $\mathcal{C}_{(0)}=$ Vec, $\mathcal{C}_{(1)}=\mathcal{C}_{p t}$ (the maximal pointed subcategory of $\mathcal{C}$ ) and $\mathcal{C}_{(n)}=\left(\mathcal{C}_{(n-1)}\right)^{c o}$ for every integer $n \geq 1$. The non-decreasing sequence of fusion subcategories of $\mathcal{C}$

$$
\mathrm{Vec}=\mathcal{C}_{(0)} \subseteq \mathcal{C}_{(1)} \subseteq \cdots \subseteq \mathcal{C}_{(n)} \subseteq \cdots
$$

is called the lower central series of $\mathcal{C}$. This definition generalizes the classical one: Let $G$ be a finite group and $\mathcal{C}=\operatorname{Rep}(G)$. Let

$$
G=C_{0}(G) \supseteq C_{1}(G) \supseteq \cdots \supseteq C_{n}(G) \supseteq \cdots
$$

be the lower central series of $G$; i.e., $C_{n}(G)=\left[G, C_{n-1}(G)\right]$ for all $n \geq 1$. Then $\mathcal{C}_{(n)}=$ $\operatorname{Rep}\left(G / C_{n}(G)\right)$.

2.3. Centralizers in braided fusion categories. Let $\mathcal{C}$ be a premodular category (i.e., a braided ribbon fusion category) with braiding $c$ and twist $\theta$ (see [BK]). By the $S$-matrix of $\mathcal{C}$ we mean a square matrix $S:=(S(X, Y))$ whose columns and rows are labeled by simple objects of $\mathcal{C}$ and the entry $S(X, Y)$ is the (quantum) trace (defined using $c$ and $\theta$ ) of $c_{Y, X} c_{X, Y}: X \otimes Y \rightarrow X \otimes Y$.

Following Müger, we will say that two objects $X, Y \in \mathcal{C}$ centralize each other if

$$
c_{Y, X} \circ c_{X, Y}=\operatorname{id}_{X \otimes Y}
$$

Assuming that categorical dimensions in $\mathcal{C}$ are positive, simple objects $X$ and $Y$ in $\mathcal{C}$ centralize each other if and only if $S(X, Y)=d(X) d(Y)$ [M1, Prop. 2.5]. All categories considered in this work have positive categorical dimensions.

Let $\mathcal{D}$ be a full (not necessarily tensor) subcategory of $\mathcal{C}$. In [M1], Müger introduced the notion of the centralizer of $\mathcal{D}$ in $\mathcal{C}$ as the fusion subcategory

$$
\mathcal{D}^{\prime}:=\left\{X \in \mathcal{C} \mid c(Y, X) \circ c(X, Y)=\operatorname{id}_{X \otimes Y}, \text { for all } Y \in \mathcal{D}\right\} .
$$

We define the Müger center of $\mathcal{C}$, denoted $\mathcal{Z}_{2}(\mathcal{C})$, to be the full fusion subcategory $\mathcal{C}^{\prime}$. The category $\mathcal{C}$ is said to be nondegenerate if $\mathcal{Z}_{2}(\mathcal{C})=$ Vec (the fusion subcategory generated by the unit object). Such a category $\mathcal{C}$ is called prime if it has no non-trivial proper nondegenerate subcategories. It was shown in [M1] that a nondegenerate braided fusion category factorizes (in general, non uniquely) into a product of prime ones.

Note that the corresponding $S$-matrix $S$ of $\mathcal{C}$ is invertible, i.e., $\mathcal{C}$ is modular, if and only if $\mathcal{C}$ is nondegenerate.

It is also known [M1] that if $\mathcal{D}$ is a fusion subcategory of a modular category $\mathcal{C}$ then $\mathcal{D}^{\prime \prime}=\mathcal{D}$ and

$$
\operatorname{dim}(\mathcal{D}) \cdot \operatorname{dim}\left(\mathcal{D}^{\prime}\right)=\operatorname{dim}(\mathcal{C})
$$

Lemma 2.1. Let $\mathcal{A}$ and $\mathcal{B}$ be full Abelian subcategories of $\mathcal{C}$ (a priori not assumed to be closed under tensor product or taking duals) centralizing each other and $\operatorname{dim}(\mathcal{A}) \cdot \operatorname{dim}(\mathcal{B})=\operatorname{dim}(\mathcal{C})$. Then $\mathcal{A}$ and $\mathcal{B}$ are fusion subcategories and $\mathcal{A}^{\prime}=\mathcal{B}$. 
Proof. Let $\tilde{\mathcal{A}}$ denote the fusion subcategory generated by $\mathcal{A}$. Note that $\mathcal{B}$ centralizes $\tilde{\mathcal{A}}$, i.e., $\mathcal{B} \subseteq(\tilde{\mathcal{A}})^{\prime}$. We have

$$
\operatorname{dim}(\mathcal{C})=\operatorname{dim}(\tilde{\mathcal{A}}) \cdot \operatorname{dim}\left((\tilde{\mathcal{A}})^{\prime}\right) \geq \operatorname{dim}(\mathcal{A}) \cdot \operatorname{dim}(\mathcal{B})=\operatorname{dim}(\mathcal{C}) .
$$

Hence, the inequality above is an equality, so $\mathcal{A}$ and $\mathcal{B}$ are fusion subcategories and $\mathcal{A}^{\prime}=$ $\mathcal{B}$.

\section{Fusion Subcategories of The QuANtum Double of A Finite Group}

3.1. The quantum double of a finite group. Let $G$ be a finite group. For any $g \in G$, let $K_{g}$ denote the conjugacy class of $G$ containing $g$. Let $R$ denote a complete set of representatives of conjugacy classes of $G$. Let $\mathcal{C}$ denote the representation category $\operatorname{Rep}(D(G))$ of the Drinfeld double of the group $G$ :

$$
\mathcal{C}:=\operatorname{Rep}(D(G)) \text {. }
$$

Remark 3.1. (i) It is well known that $\mathcal{C}$ is equivalent, as a braided fusion category, to the category of $G$-equivariant vector bundles on $G$, where $G$ acts on itself by conjugation.

(ii) The category $\mathcal{C}$ contains the category $\operatorname{Rep}(G)$ of representations of $G$ as a fusion subcategory. Namely, $\operatorname{Rep}(G)$ is identified with the category of $G$-equivariant bundles supported on the identity element of $G$.

The set of isomorphism classes of simple objects of $\mathcal{C}$ is identified with the following set:

$$
\Gamma:=\left\{(a, \chi) \mid a \in R \text { and } \chi \text { is an irreducible character of } C_{G}(a)\right\} .
$$

The categorical dimension of the simple object $(a, \chi)$ is

$$
d(a, \chi)=\left|K_{a}\right| \operatorname{deg} \chi=\frac{|G|}{\left|C_{G}(a)\right|} \operatorname{deg} \chi .
$$

It is well known that $\mathcal{C}$ is a modular category $\mathrm{AC1}$. Its $S$-matrix $S$ and twist $\theta$ are given by the following formulas :

$$
\begin{aligned}
S\left((a, \chi),\left(b, \chi^{\prime}\right)\right) & =\frac{|G|}{\left|C_{G}(a)\right|\left|C_{G}(b)\right|} \sum_{g \in G(a, b)} \bar{\chi}\left(g b g^{-1}\right) \bar{\chi}^{\prime}\left(g^{-1} a g\right), \\
\theta(a, \chi) & =\frac{\chi(a)}{\operatorname{deg} \chi},
\end{aligned}
$$

for all $(a, \chi),\left(b, \chi^{\prime}\right) \in \Gamma$, where $G(a, b)=\left\{g \in G \mid a g b g^{-1}=g b g^{-1} a\right\}$ and $\bar{\chi}$ denotes the character conjugate to $\chi$. For more details we refer the reader to [BK].

Note that the categorical dimensions (4) of objects of $\mathcal{C}$ are defined using the braiding on $\mathcal{C}$ and the above twist $\theta$.

Remark 3.2. It is known that the entries of the $S$-matrix lie in a cyclotomic field. Also, the values of characters of a finite group are sums of roots of unity. So we may assume that all scalars appearing herein are complex numbers; in particular, complex conjugation and absolute values make sense.

The following lemma was proved in [NN, Lemma 3.1]. 
Lemma 3.3. Two objects $(a, \chi),\left(b, \chi^{\prime}\right) \in \Gamma$ centralize each other if and only if the following conditions hold:

(i) The conjugacy classes $K_{a}, K_{b}$ commute element-wise,

(ii) $\chi\left(g b g^{-1}\right) \chi^{\prime}\left(g^{-1} a g\right)=\operatorname{deg} \chi \operatorname{deg} \chi^{\prime}$, for all $g \in G$.

We generalize this result in the next lemma.

Lemma 3.4. For any two objects $(a, \chi),\left(b, \chi^{\prime}\right) \in \Gamma,\left|S\left((a, \chi),\left(b, \chi^{\prime}\right)\right)\right|=d(a, \chi) d\left(b, \chi^{\prime}\right)$ if and only if the following conditions hold:

(i) The conjugacy classes $K_{a}, K_{b}$ commute element-wise,

(ii) $\left|\chi\left(g b g^{-1}\right)\right|=\operatorname{deg} \chi,\left|\chi^{\prime}\left(g^{-1} a g\right)\right|=\operatorname{deg} \chi^{\prime}$, and $\chi\left(g b g^{-1}\right) \chi^{\prime}\left(g^{-1} a g\right)=\chi(b) \chi^{\prime}(a)$, for all $g \in G$.

Proof. The condition $\left|S\left((a, \chi),\left(b, \chi^{\prime}\right)\right)\right|=d(a, \chi) d\left(b, \chi^{\prime}\right)$ is equivalent to the condition

$$
\left|\sum_{g \in G(a, b)} \chi\left(g b g^{-1}\right) \chi^{\prime}\left(g^{-1} a g\right)\right|=|G| \operatorname{deg} \chi \operatorname{deg} \chi^{\prime},
$$

where $G(a, b)=\left\{g \in G \mid a g b g^{-1}=g b g^{-1} a\right\}$. It is clear that if the two conditions in the statement of the lemma hold, then (5) holds.

Now suppose that (5) holds. We will show that this implies the two conditions in the statement of the lemma. We have

$$
\begin{aligned}
|G| \operatorname{deg} \chi \operatorname{deg} \chi^{\prime} & =\left|\sum_{g \in G(a, b)} \chi\left(g b g^{-1}\right) \chi^{\prime}\left(g^{-1} a g\right)\right| \\
& \leq \sum_{g \in G(a, b)}\left|\chi\left(g b g^{-1}\right)\right|\left|\chi^{\prime}\left(g^{-1} a g\right)\right| \\
& \leq|G| \operatorname{deg} \chi \operatorname{deg} \chi^{\prime} .
\end{aligned}
$$

So $\sum_{g \in G(a, b)}\left|\chi\left(g b g^{-1}\right)\right|\left|\chi^{\prime}\left(g^{-1} a g\right)\right|=|G| \operatorname{deg} \chi \operatorname{deg} \chi^{\prime}$. Since

$$
|G(a, b)| \leq|G|,\left|\chi\left(g b g^{-1}\right)\right| \leq \operatorname{deg} \chi, \text { and }\left|\chi^{\prime}\left(g^{-1} a g\right)\right| \leq \operatorname{deg} \chi^{\prime},
$$

we must have $G(a, b)=G,\left|\chi\left(g b g^{-1}\right)\right|=\operatorname{deg} \chi$, and $\left|\chi^{\prime}\left(g^{-1} a g\right)\right|=\operatorname{deg} \chi^{\prime}$. The equality $G(a, b)=G$ implies that the conjugacy classes $K_{a}, K_{b}$ commute element-wise. Since $\left|\chi\left(g b g^{-1}\right)\right|=\operatorname{deg} \chi$ and $\left|\chi^{\prime}\left(g^{-1} a g\right)\right|=\operatorname{deg} \chi^{\prime}$, there exist roots of unity $\alpha_{g}$ and $\beta_{g}$ such that $\chi\left(g b g^{-1}\right)=\alpha_{g} \operatorname{deg} \chi$ and $\chi^{\prime}\left(g^{-1} a g\right)=\beta_{g} \operatorname{deg} \chi^{\prime}$, for all $g \in G$. Substitute in (5) to obtain the equation

$$
\left|\sum_{g \in G} \alpha_{g} \beta_{g}\right|=|G| .
$$

Note that (6) holds if and only if $\alpha_{g} \beta_{g}=\alpha_{e} \beta_{e}$, for all $g \in G$. This is equivalent to saying that $\chi\left(g b g^{-1}\right) \chi^{\prime}\left(g^{-1} a g\right)=\chi(b) \chi^{\prime}(a)$, for all $g \in G$, and the lemma is proved.

Note 3.5. The following special cases of Lemma 3.4 will be used later.

(i) $\left|S\left((e, \chi),\left(b, \chi^{\prime}\right)\right)\right|=d(e, \chi) d\left(b, \chi^{\prime}\right)$ if and only if $\left.\chi\right|_{[G, b]}=\operatorname{deg} \chi$, where

$$
[G, b]=\left\langle g b g_{6}^{-1} b^{-1} \mid g \in G\right\rangle .
$$


(ii) $\left|S\left((a, 1),\left(b, \chi^{\prime}\right)\right)\right|=d(a, 1) d\left(b, \chi^{\prime}\right)$ if and only if the conjugacy classes $K_{a}, K_{b}$ commute element-wise and $\left.\chi^{\prime}\right|_{[G, a]}=\operatorname{deg} \chi^{\prime}$.

3.2. Canonical data determined by a fusion subcategory of $\operatorname{Rep}(\mathbf{D}(\mathbf{G}))$. Let $\mathcal{D}$ be a fusion subcategory of $\mathcal{C}$. There are two canonical normal subgroups of $G$ determined by $\mathcal{D}$. First, let

$$
K_{\mathcal{D}}:=\left\{g a g^{-1} \mid g \in G \text { and }(a, \chi) \in \mathcal{D} \cap \Gamma \text { for some } \chi\right\}
$$

be the support of $\mathcal{D}$. That is, $K_{\mathcal{D}}$ is a minimal subgroup of $G$ with the property that every vector bundle in $\mathcal{D}$ is supported on $K_{\mathcal{D}}$.

Second, let $H_{\mathcal{D}}$ be the normal subgroup of $G$ such that $\mathcal{D} \cap \operatorname{Rep}(G)=\operatorname{Rep}\left(G / H_{\mathcal{D}}\right)$. Equivalently,

$$
H_{\mathcal{D}}:=\bigcap_{\chi:(e, \chi) \in \mathcal{D}} \operatorname{Ker}(\chi),
$$

where $\operatorname{Ker}(\chi)=\{g \in G \mid \chi(g)=\operatorname{deg} \chi\}$ is the kernel of the character $\chi$.

Proposition 3.6. Let $\mathcal{D}$ be a fusion subcategory of $\mathcal{C}$. Then $H_{\mathcal{D}^{\prime}}=K_{\mathcal{D}}, K_{\mathcal{D}^{\prime}}=H_{\mathcal{D}}$, and the subgroups $K_{\mathcal{D}}$ and $H_{\mathcal{D}}$ centralize each other.

Proof. Consider a simple object $(e, \chi)$ of $\operatorname{Rep}(G)$. By Lemma 3.3 it centralizes $\mathcal{D}$ if and only if $\chi(a)=\operatorname{deg} \chi$ for all $a \in K_{\mathcal{D}}$. Thus, $\mathcal{D}^{\prime} \cap \operatorname{Rep}(G)=\operatorname{Rep}\left(G / K_{\mathcal{D}}\right)$, which is the first equality.

The second equality follows by replacing $\mathcal{D}$ with $\mathcal{D}^{\prime}$ and using Müger's double centralizer theorem $\left(\mathcal{D}^{\prime \prime}=\mathcal{D}\right)$ [M1]. By Lemma 3.3, $K_{\mathcal{D}}$ and $K_{\mathcal{D}^{\prime}}$ centralize each other.

Lemma 3.7. Let $\mathcal{E}$ be a fusion subcategory of $\operatorname{Rep}(D(G))$ and let $(a, \chi) \in \mathcal{E} \cap \Gamma$. Suppose $\chi^{\prime}$ is an irreducible character of $C_{G}(a)$ such that $\frac{\left.\chi^{\prime}\right|_{\mathcal{E}^{\prime}}}{\operatorname{deg} \chi^{\prime}}=\frac{\left.\chi\right|_{\mathcal{E}^{\prime}}}{\operatorname{deg} \chi}$, where $K_{\mathcal{E}^{\prime}}$ is the support of $\mathcal{E}^{\prime}$. Then $\left(a, \chi^{\prime}\right) \in \mathcal{E}$.

Proof. Let us show that $\left(a, \chi^{\prime}\right)$ centralizes $\mathcal{E}^{\prime}$. Pick any $\left(b, \chi^{\prime \prime}\right) \in \mathcal{E}^{\prime} \cap \Gamma$. To see that $\left(a, \chi^{\prime}\right)$ and $\left(b, \chi^{\prime \prime}\right)$ centralize each other we only need to check that Condition (ii) of Lemma 3.3 is satisfied. We have

$$
\frac{\chi^{\prime}\left(g b g^{-1}\right)}{\operatorname{deg} \chi^{\prime}} \frac{\chi^{\prime \prime}\left(g^{-1} a g\right)}{\operatorname{deg} \chi^{\prime \prime}}=\frac{\chi\left(g b g^{-1}\right)}{\operatorname{deg} \chi} \frac{\chi^{\prime \prime}\left(g^{-1} a g\right)}{\operatorname{deg} \chi^{\prime \prime}}=1
$$

for all $g \in G$. The first equality above is due to the equality $\frac{\left.\chi^{\prime}\right|_{K_{\mathcal{E}^{\prime}}}}{\operatorname{deg} \chi^{\prime}}=\frac{\left.\chi\right|_{K_{\mathcal{E}^{\prime}}}}{\operatorname{deg} \chi}$, while the second equality is due to the fact that $\left(a, \chi^{\prime}\right)$ and $\left(b, \chi^{\prime \prime}\right)$ centralize each other. Therefore, $\left(a, \chi^{\prime}\right)$ centralizes $\mathcal{E}^{\prime}$, i.e., $\left(a, \chi^{\prime}\right) \in \mathcal{E}^{\prime \prime}=\mathcal{E}$ and the lemma is proved.

Let us define a pairing

$$
B_{\mathcal{D}}: K_{\mathcal{D}} \times H_{\mathcal{D}}=K_{\mathcal{D}} \times K_{\mathcal{D}^{\prime}} \rightarrow k^{\times}
$$

as follows. Let $(a, \chi) \in \mathcal{D} \cap \Gamma$, and

$$
B_{\mathcal{D}}\left(g^{-1} a g, h\right):=\frac{\chi\left(g h g^{-1}\right)}{\operatorname{deg} \chi},
$$


for all $g \in G, h \in H_{\mathcal{D}}$. Let $\left(b, \chi^{\prime}\right) \in \mathcal{D}^{\prime} \cap \Gamma$, so that $\left(b, \chi^{\prime}\right)$ centralizes $(a, \chi)$. Then

$$
\frac{\chi\left(g b g^{-1}\right)}{\operatorname{deg} \chi}=\left(\frac{\chi^{\prime}\left(g^{-1} a g\right)}{\operatorname{deg} \chi^{\prime}}\right)^{-1}
$$

for all $g \in G$. The equation (10) shows that the pairing does not depend on the choice of $\chi$. By its definition, $B_{\mathcal{D}}$ is $G$-invariant. Also note that

$$
B_{\mathcal{D}}\left(k k^{\prime}, h\right)=B_{\mathcal{D}}(k, h) B_{\mathcal{D}}\left(k^{\prime}, h\right) \quad \text { and } \quad B_{\mathcal{D}}\left(k, h h^{\prime}\right)=B_{\mathcal{D}}(k, h) B_{\mathcal{D}}\left(k, h^{\prime}\right)
$$

for all $k, k^{\prime} \in K_{\mathcal{D}}$ and $h, h^{\prime} \in H_{\mathcal{D}}=K_{\mathcal{D}^{\prime}}$; the latter equality is immediate from the definition of $B_{\mathcal{D}}$, while the former follows from equation (10) which gives an alternative definition of $B_{\mathcal{D}}$.

Thus, to every fusion subcategory $\mathcal{D} \subseteq \mathcal{C}$ we associate a canonical triple $\left(K_{\mathcal{D}}, H_{\mathcal{D}}, B_{\mathcal{D}}\right)$, where $K_{\mathcal{D}}$ and $H_{\mathcal{D}}$ are normal subgroups of $G$ centralizing each other and $B_{\mathcal{D}}: K_{\mathcal{D}} \times H_{\mathcal{D}} \rightarrow k^{\times}$ is a $G$-invariant bicharacter.

3.3. Construction of a fusion subcategory of $\operatorname{Rep}(D(G))$. Suppose we have two normal subgroups $K$ and $H$ of $G$ that centralize each other, and a $G$-invariant bicharacter $B$ : $K \times H \rightarrow k^{\times}$. Define

$$
\begin{aligned}
\mathcal{S}(K, H, B):= & \text { full Abelian subcategory of } \mathcal{C} \text { generated by } \\
& \left\{(a, \chi) \in \Gamma \mid \begin{array}{c}
a \in K \cap R \text { and } \chi \text { is an irreducible character of } C_{G}(a) \\
\text { such that } \chi(h)=B(a, h) \operatorname{deg} \chi, \text { for all } h \in H
\end{array}\right\} .
\end{aligned}
$$

Remark 3.8. (i) As we mentioned in $\operatorname{Remark} 3.1$, $\operatorname{Rep}(D(G))$ is identified with the category of $G$-equivariant bundles on $G$. The above subcategory $\mathcal{S}(K, H, B)$ is identified with the subcategory of bundles supported on $K$ whose $G$-equivariant structure restricts to $H$ as follows: The action of $h \in H$ on the fiber corresponding to $a \in K$ is the scalar multiplication by $B(a, h)$. That $K$ is indeed the support of $\mathcal{S}(K, H, B)$ follows from the next lemma.

(ii) Note that $\mathcal{S}(G,\{e\}, 1)=\operatorname{Rep}(D(G))$, while $\mathcal{S}(\{e\}, G, 1)$ is the trivial fusion subcategory of $\operatorname{Rep}(D(G))$.

The following lemma was proved in [NN, Lemma 3.2].

Lemma 3.9. Let $E$ be a normal subgroup of a finite group $F$. Let $\operatorname{Irr}(F)$ denote the set of irreducible characters of $F$. Let $\rho$ be an $F$-invariant character of $E$ of degree 1 . Then

$$
\sum_{\chi \in \operatorname{Irr}(F):\left.\chi\right|_{E}=(\operatorname{deg} \chi) \rho}(\operatorname{deg} \chi)^{2}=[F: E] .
$$

Recall that by the dimension of a full Abelian subcategory of $\mathcal{C}$ we mean the sum of squares of dimensions of its simple objects.

Lemma 3.10. The dimension of the subcategory $\mathcal{S}(K, H, B)$ is $|K|[G: H]$. 
Proof. We compute

$$
\begin{aligned}
\operatorname{dim}(\mathcal{S}(K, H, B)) & =\sum_{(a, \chi) \in \mathcal{S}(K, H, B) \cap \Gamma} \operatorname{dim}((a, \chi))^{2} \\
& =\sum_{a \in K \cap R}\left|K_{a}\right|^{2} \sum_{\chi:(a, \chi) \in \mathcal{S}(K, H, B) \cap \Gamma}(\operatorname{deg} \chi)^{2} \\
& =\sum_{a \in K \cap R}\left|K_{a}\right|^{2}\left[C_{G}(a): H\right]=|K|[G: H],
\end{aligned}
$$

where the third equality above is explained as follows. Fix $a \in K \cap R$ and observe that $B(a, \cdot)$ is a $C_{G}(a)$-invariant character of $H$ of degree 1 and then apply Lemma 3.9.

Given a bicharacter $B: K \times H \rightarrow k^{\times}$let us define $B^{\text {op }}: H \times K \rightarrow k^{\times}$by $B^{\text {op }}(h, k)=$ $B(k, h)$ for all $k \in K, h \in H$.

Lemma 3.11. $\mathcal{S}(K, H, B)$ is a fusion subcategory of $\mathcal{C}$ and $\mathcal{S}(K, H, B)^{\prime}=\mathcal{S}\left(H, K,\left(B^{\mathrm{op}}\right)^{-1}\right)$.

Proof. First, we show that the full Abelian subcategories $\mathcal{S}(K, H, B), \mathcal{S}\left(H, K,\left(B^{\mathrm{op}}\right)^{-1}\right) \subseteq \mathcal{C}$ centralize each other. Let $(a, \chi) \in \mathcal{S}(K, H, B)$ and $\left(b, \chi^{\prime}\right) \in \mathcal{S}\left(H, K,\left(B^{\mathrm{op}}\right)^{-1}\right)$. Since $K$ and $H$ centralize each other, in order to show that $(a, \chi)$ and $\left(b, \chi^{\prime}\right)$ centralize each other, it only remains to check that condition (ii) of Lemma 3.3 holds. We have

$$
\frac{\chi\left(g b g^{-1}\right)}{\operatorname{deg} \chi} \frac{\chi^{\prime}\left(g^{-1} a g\right)}{\operatorname{deg} \chi^{\prime}}=B\left(a, g b g^{-1}\right)\left(B^{\mathrm{op}}\right)^{-1}\left(b, g^{-1} a g\right)=B\left(a, g b g^{-1}\right) B\left(g^{-1} a g, b\right)^{-1}=1,
$$

for all $g \in G$. The first equality above is by definition of $\mathcal{S}(K, H, B)$ and $\mathcal{S}\left(H, K,\left(B^{\text {op }}\right)^{-1}\right)$, while the last equality is due to $G$-invariance of $B$. Therefore, condition (ii) of Lemma 3.3 holds and it follows that $\mathcal{S}(K, H, B)$ and $\mathcal{S}\left(H, K,\left(B^{\mathrm{op}}\right)^{-1}\right)$ centralize each other.

By Lemma 3.10, $\operatorname{dim}(\mathcal{S}(K, H, B)) \cdot \operatorname{dim}\left(\mathcal{S}\left(H, K,\left(B^{\text {op }}\right)^{-1}\right)=|K|[G: H] \cdot|H|[G: K]=\right.$ $|G|^{2}=\operatorname{dim}(\mathcal{C})$. Since $\mathcal{S}(K, H, B)$ and $\mathcal{S}\left(H, K,\left(B^{\circ \mathrm{op}}\right)^{-1}\right)$ centralize each other it follows from Lemma 2.1 that $\mathcal{S}(K, H, B)$ is a fusion subcategory and $\mathcal{S}(K, H, B)^{\prime}=\mathcal{S}\left(H, K,\left(B^{\mathrm{op}}\right)^{-1}\right)$.

Theorem 3.12. The assignments $\mathcal{D} \mapsto\left(K_{\mathcal{D}}, H_{\mathcal{D}}, B_{\mathcal{D}}\right)$ and $(K, H, B) \mapsto \mathcal{S}(K, H, B)$ are inverses of each other. Thus, there is a bijection between the set of fusion subcategories of $\mathcal{C}$ and triples $(K, H, B)$, where $K, H \subseteq G$ are normal subgroups of $G$ centralizing each other and $B: K \times H \rightarrow k^{\times}$is a $G$-invariant bicharacter.

Proof. First, we show that $\left(K_{\mathcal{S}(K, H, B)}, H_{\mathcal{S}(K, H, B)}, B_{\mathcal{S}(K, H, B)}\right)=(K, H, B)$. As mentioned in Remark 3.8, it follows from Lemma 3.9 that $K_{\mathcal{S}(K, H, B)}=K$. By Lemma 3.11, $H_{\mathcal{S}(K, H, B)}$ is the support of $\mathcal{S}(K, H, B)^{\prime}$ and so $H_{\mathcal{S}(K, H, B)}=H$. For any $a \in K \cap R, h \in H$, we have $B_{\mathcal{S}(K, H, B)}(a, h)=\frac{\chi(h)}{\operatorname{deg} \chi}$, where $(a, \chi) \in \mathcal{S}(K, H, B) \cap \Gamma$. Since $(a, \chi) \in \mathcal{S}(K, H, B)$, we have $\chi(h)=B(a, h) \operatorname{deg} \chi$. Therefore, $B_{\mathcal{S}(K, H, B)}(a, h)=B(a, h)$ and the $G$-invariance of the two bicharacters in question imply that $B_{\mathcal{S}(K, H, B)}=B$.

Second, we show that $\mathcal{S}\left(K_{\mathcal{D}}, H_{\mathcal{D}}, B_{\mathcal{D}}\right)=\mathcal{D}$. Pick any $(a, \chi) \in \mathcal{S}\left(K_{\mathcal{D}}, H_{\mathcal{D}}, B_{\mathcal{D}}\right)$. Then for all $h \in H_{\mathcal{D}}$,

$$
\frac{\chi(h)}{\operatorname{deg} \chi}=B_{\mathcal{D}}(a, h)=\frac{\chi^{\prime}(h)}{\operatorname{deg} \chi^{\prime}},
$$

where $\left(a, \chi^{\prime}\right) \in \mathcal{D}$. Here the first equality follows from the definition of $\mathcal{S}\left(K_{\mathcal{D}}, H_{\mathcal{D}}, B_{\mathcal{D}}\right)$ (11), while the second equality follows from the definition of $B_{\mathcal{D}}(9)$. Therefore, $\frac{\left.\chi\right|_{\mathcal{D}^{\prime}}}{\operatorname{deg} \chi}=$ 
$\frac{\left.\chi^{\prime}\right|_{\mathcal{D}^{\prime}}}{\operatorname{deg} \chi^{\prime}}$ and it follows from Lemma 3.7 that $(a, \chi) \in \mathcal{D}$. So, $\mathcal{S}\left(K_{\mathcal{D}}, H_{\mathcal{D}}, B_{\mathcal{D}}\right) \subseteq \mathcal{D}$. Similarly, $\mathcal{S}\left(K_{\mathcal{D}^{\prime}}, H_{\mathcal{D}^{\prime}}, B_{\mathcal{D}^{\prime}}\right)=\mathcal{S}\left(H_{\mathcal{D}}, K_{\mathcal{D}}, B_{\mathcal{D}^{\prime}}\right) \subseteq \mathcal{D}^{\prime}$. Using Lemma 3.10 we have

$\operatorname{dim}(\mathcal{C})=|G|^{2}=\operatorname{dim}\left(\mathcal{S}\left(K_{\mathcal{D}}, H_{\mathcal{D}}, B_{\mathcal{D}}\right)\right) \cdot \operatorname{dim}\left(\mathcal{S}\left(H_{\mathcal{D}}, K_{\mathcal{D}}, B_{\mathcal{D}^{\prime}}\right)\right) \leq \operatorname{dim}(\mathcal{D}) \operatorname{dim}\left(\mathcal{D}^{\prime}\right)=\operatorname{dim}(\mathcal{C})$.

Hence, $\operatorname{dim}\left(\mathcal{S}\left(K_{\mathcal{D}}, H_{\mathcal{D}}, B_{\mathcal{D}}\right)\right)=\operatorname{dim}(\mathcal{D})$ and so $\mathcal{S}\left(K_{\mathcal{D}}, H_{\mathcal{D}}, B_{\mathcal{D}}\right)=\mathcal{D}$.

\section{Some invariants And the lattice of Fusion subcategories of $\operatorname{Rep}(D(G))$}

4.1. The lattice. Let $G$ be a finite group. Let $K, H, K^{\prime}$, and $H^{\prime}$ be normal subgroups of $G$ such that $K$ and $H$ centralize each other and $K^{\prime}$ and $H^{\prime}$ centralize each other. Let $B: K \times H: \rightarrow k^{\times}$and $B^{\prime}: K^{\prime} \times H^{\prime}: \rightarrow k^{\times}$be $G$-invariant bicharacters.

Proposition 4.1. $\mathcal{S}(K, H, B) \subseteq \mathcal{S}\left(K^{\prime}, H^{\prime}, B^{\prime}\right)$ if and only if $K \subseteq K^{\prime}, H^{\prime} \subseteq H$, and $\left.B\right|_{K \times H^{\prime}}=\left.B^{\prime}\right|_{K \times H^{\prime}}$.

Proof. The statement follows from the definitions and Remark 3.8

Define a homomorphism

$$
\varphi_{B, B^{\prime}}: K \cap K^{\prime} \rightarrow \widehat{H \cap H^{\prime}} \quad \text { by }\left.\left.\quad a \mapsto B(a, \cdot)\right|_{H \cap H^{\prime}}\left(B^{\prime}\right)^{-1}(a, \cdot)\right|_{H \cap H^{\prime}},
$$

where $\widehat{H \cap H^{\prime}}$ denotes the group of group homomorphisms from $H \cap H^{\prime}$ to $k^{\times}$. Also, define a $G$-invariant bicharacter

$$
\psi_{B, B^{\prime}}: \operatorname{ker} \varphi_{B, B^{\prime}} \times H H^{\prime} \rightarrow k^{\times} \quad \text { by } \quad\left(a, h h^{\prime}\right) \mapsto B(a, h) B^{\prime}\left(a, h^{\prime}\right) .
$$

Proposition 4.2. $\quad$ (i) $\mathcal{S}(K, H, B) \cap \mathcal{S}\left(K^{\prime}, H^{\prime}, B^{\prime}\right)=\mathcal{S}\left(\operatorname{ker} \varphi_{B, B^{\prime}}, H H^{\prime}, \psi_{B, B^{\prime}}\right)$.

(ii) $\mathcal{S}(K, H, B) \vee \mathcal{S}\left(K^{\prime}, H^{\prime}, B^{\prime}\right)=\mathcal{S}\left(K K^{\prime}, \operatorname{ker} \varphi_{B^{\mathrm{op}},\left(B^{\prime}\right)^{\mathrm{op}}},\left(\psi_{B^{\mathrm{op}},\left(B^{\prime}\right)^{\mathrm{op}}}\right)^{\mathrm{op}}\right)$.

Proof. (i) First, we show that $\mathcal{S}(K, H, B) \cap \mathcal{S}\left(K^{\prime}, H^{\prime}, B^{\prime}\right) \subseteq \mathcal{S}\left(\operatorname{ker} \varphi_{B, B^{\prime}}, H H^{\prime}, \psi_{B, B^{\prime}}\right)$. Let $(a, \chi)$ be any simple object of $\mathcal{S}(K, H, B) \cap \mathcal{S}\left(K^{\prime}, H^{\prime}, B^{\prime}\right)$. By definition, $a \in K \cap K^{\prime} \cap R$ and $\chi$ is an irreducible character of $C_{G}(a)$ such that

$$
\left.\chi\right|_{H}=B(a, \cdot) \operatorname{deg} \chi \text { and }\left.\chi\right|_{H^{\prime}}=B^{\prime}(a, \cdot) \operatorname{deg} \chi .
$$

It follows that $\left.B(a, \cdot)\right|_{H \cap H^{\prime}}=\left.B^{\prime}(a, \cdot)\right|_{H \cap H^{\prime}}$, so that $a \in \operatorname{ker} \varphi_{B, B^{\prime}}$. Also,

$$
\chi\left(h h^{\prime}\right)=B(a, h) B^{\prime}\left(a, h^{\prime}\right) \operatorname{deg} \chi=\psi_{B, B^{\prime}}\left(a, h h^{\prime}\right) \operatorname{deg} \chi,
$$

for all $h \in H, h^{\prime} \in H^{\prime}$. Therefore, $(a, \chi) \in \mathcal{S}\left(\operatorname{ker} \varphi_{B, B^{\prime}}, H H^{\prime}, \psi_{B, B^{\prime}}\right)$, so $\mathcal{S}(K, H, B) \cap$ $\mathcal{S}\left(K^{\prime}, H^{\prime}, B^{\prime}\right) \subseteq \mathcal{S}\left(\operatorname{ker} \varphi_{B, B^{\prime}}, H H^{\prime}, \psi_{B, B^{\prime}}\right)$.

Next, we show that $\mathcal{S}\left(\operatorname{ker} \varphi_{B, B^{\prime}}, H H^{\prime}, \psi_{B, B^{\prime}}\right) \subseteq \mathcal{S}(K, H, B) \cap \mathcal{S}\left(K^{\prime}, H^{\prime}, B^{\prime}\right)$. Let $(a, \chi)$ be a simple object in $\mathcal{S}\left(\operatorname{ker} \varphi_{B, B^{\prime}}, H H^{\prime}, \psi_{B, B^{\prime}}\right)$. By definition, $a \in \operatorname{ker} \varphi_{B, B^{\prime}} \cap R \subseteq K \cap K^{\prime} \cap R$ and $\chi$ is an irreducible character of $C_{G}(a)$ such that

$$
\left.\chi\right|_{H H^{\prime}}=\psi_{B, B^{\prime}}(a, \cdot) \operatorname{deg} \chi .
$$

If $h \in H$, this implies

$$
\chi(h)=\psi_{B, B^{\prime}}(a, h) \operatorname{deg} \chi=B(a, h) B^{\prime}(a, e) \operatorname{deg} \chi=B(a, h) \operatorname{deg} \chi .
$$

Similarly, if $h^{\prime} \in H^{\prime}$, then $\chi\left(h^{\prime}\right)=B^{\prime}\left(a, h^{\prime}\right) \operatorname{deg} \chi$. By definition, $(a, \chi) \in \mathcal{S}(K, H, B) \cap$ $\mathcal{S}\left(K^{\prime}, H^{\prime}, B^{\prime}\right)$, so $\mathcal{S}\left(\operatorname{ker} \varphi_{B, B^{\prime}}, H H^{\prime}, \psi_{B, B^{\prime}}\right) \subseteq \mathcal{S}(K, H, B) \cap \mathcal{S}\left(K^{\prime}, H^{\prime}, B^{\prime}\right)$ and the proposition is proved. 
(ii) We have

$$
\begin{aligned}
& \mathcal{S}(K, H, B) \vee \mathcal{S}\left(K^{\prime}, H^{\prime}, B^{\prime}\right)=\left(\mathcal{S}(K, H, B)^{\prime} \cap \mathcal{S}\left(K^{\prime}, H^{\prime}, B^{\prime}\right)^{\prime}\right)^{\prime} \\
& =\left(\mathcal{S}\left(H, K,\left(B^{\mathrm{op}}\right)^{-1}\right) \cap \mathcal{S}\left(H^{\prime}, K^{\prime},\left(\left(B^{\prime}\right)^{\mathrm{op}}\right)^{-1}\right)\right)^{\prime}
\end{aligned}
$$

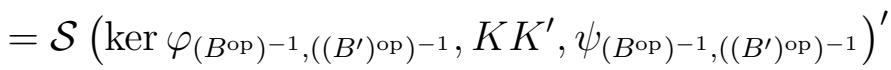

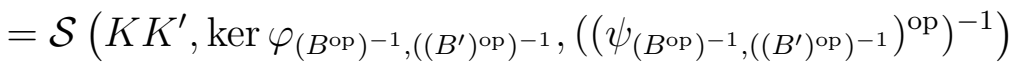

$$
\begin{aligned}
& \left.=\mathcal{S}\left(K K^{\prime}, \operatorname{ker} \varphi_{B^{\mathrm{op}},\left(B^{\prime}\right)}{ }^{\mathrm{op}}\right),\left(\psi_{B^{\mathrm{op}},\left(B^{\prime}\right)^{\mathrm{op}}}\right)^{\mathrm{op}}\right) \text {. }
\end{aligned}
$$

The first equality above is due to [M1, Lemma 2.8 and Theorem 3.2(i)], the last equality follows from a direct calculation, and the other equalities follow from either Lemma 3.11 or part (i).

A braided tensor category $\mathcal{C}$ is said to be symmetric if the square of the braiding is the identity $[\mathrm{BK}]$. A fusion subcategory $\mathcal{D}$ of a premodular category $\mathcal{C}$ with positive categorical dimensions is said to be isotropic if the twist of $\mathcal{C}$ restricts to the identity on $\mathcal{D}$ [DGNO]. By Deligne's theorem [D] an isotropic subcategory $\mathcal{D} \subset \mathcal{C}$ is Tannakian, i.e., it is equivalent to the representation category of a finite group as a symmetric fusion category. An isotropic subcategory $\mathcal{D} \subseteq \mathcal{C}$ is said to be Lagrangian if $\mathcal{D}^{\prime}=\mathcal{D}$, or equivalently $(\operatorname{dim}(\mathcal{D}))^{2}=\operatorname{dim}(\mathcal{C})$. Recall that a bicharacter $f: L \times L \rightarrow k^{\times}$on an abelian group $L$ is alternating if $f(x, x)=1$, for all $x \in L$.

Proposition 4.3. The fusion subcategory $\mathcal{S}(K, H, B) \subseteq \operatorname{Rep}(D(G))$ is

(i) symmetric if and only if $K \subseteq H$ and $B\left(k_{1}, k_{2}\right) B\left(k_{2}, k_{1}\right)=1$, for all $k_{1}, k_{2} \in K$,

(ii) isotropic if and only if $K \subseteq H$ and $\left.B\right|_{K \times K}$ is alternating,

(iii) Lagrangian if and only if $K=H$ and $B$ is alternating.

Proof. (i) $\mathcal{S}(K, H, B)$ is symmetric if and only if $\mathcal{S}(K, H, B) \subseteq \mathcal{S}(K, H, B)^{\prime}$. By Lemma 3.11 and Proposition 4.1, this is true if and only if $K \subseteq H$ and $\left.B\right|_{K \times K}=\left.\left(B^{\mathrm{op}}\right)^{-1}\right|_{K \times K}$, as stated.

(ii) Suppose $K \subseteq H$ and $\left.B\right|_{K \times K}$ is alternating. In particular, this implies $B(a, a)=1$ for all $a \in K \cap R$, so that

$$
\theta(a, \chi)=\frac{\chi(a)}{\operatorname{deg} \chi}=B(a, a)=1
$$

for all $(a, \chi) \in \mathcal{S}(K, H, B) \cap \Gamma$. That is, $\mathcal{S}(K, H, B)$ is isotropic.

Conversely, assume $\mathcal{S}(K, H, B)$ is isotropic, so in particular $\mathcal{S}(K, H, B)$ is symmetric. Therefore, by (ii), $K \subseteq H$ and $B\left(k_{1}, k_{2}\right) B\left(k_{2}, k_{1}\right)=1$, for all $k_{1}, k_{2} \in K$. It remains to show that $B(k, k)=1$, for all $k \in K$; to this end, pick any $(a, \chi) \in \mathcal{S}(K, H, B) \cap \Gamma$ and observe that

$$
B(a, a)=\frac{\chi(a)}{\operatorname{deg} \chi}=\theta(a, \chi)=1
$$

where the last equality is due to the definition of isotropic. Normality of $K$ and $G$-invariance of $B$ together imply that $B(k, k)=1$, for all $k \in K$, as desired.

(iii) This was proved in [NN].

Recall that the notions of Müger's center $\mathcal{Z}_{2}$, nondegenerate and prime braided fusion categories were defined in Section [2.3. Also, recall that a symmetric bicharacter $f: L \times L \rightarrow$ $k^{\times}$on a group $L$ is nondegenerate if $\{x \in L \mid f(x, y)=1$ for all $y \in L\}=\{e\}$.

Proposition 4.4. $\mathcal{Z}_{2}(\mathcal{S}(K, H, B))=\mathcal{S}\left(\operatorname{ker} \varphi_{B,\left(B^{\mathrm{op}}\right)^{-1}}, H K, \psi_{B,\left(B^{\mathrm{op}}\right)^{-1}}\right)$. 
Proof. Using Lemma 3.11 and Proposition 4.2 (i), we have

$$
\begin{aligned}
\mathcal{Z}_{2}(\mathcal{S}(K, H, B)) & =\mathcal{S}(K, H, B) \cap \mathcal{S}(K, H, B)^{\prime} \\
& =\mathcal{S}(K, H, B) \cap \mathcal{S}\left(H, K,\left(B^{\mathrm{op}}\right)^{-1}\right) \\
& =\mathcal{S}\left(\operatorname{ker} \varphi_{B,\left(B^{\text {op }}\right)^{-1}}, H K, \psi_{B,\left(B^{\text {op }}\right)^{-1}}\right) .
\end{aligned}
$$

Proposition 4.5. (i) The fusion subcategory $\mathcal{S}(K, H, B) \subseteq \operatorname{Rep}(D(G))$ is nondegenerate if and only if $H K=G$ and the symmetric bicharacter $\left.B B^{\mathrm{op}}\right|_{(K \cap H) \times(K \cap H)}$ is nondegenerate.

(ii) $\operatorname{Rep}(D(G))$ is prime if and only if there is no triple $(K, H, B)$, where $K$ and $H$ are normal subgroups of $G$ that centralize each other, $(G,\{e\}) \neq(K, H) \neq(\{e\}, G)$, $H K=G$, and $B$ is a $G$-invariant bicharacter on $K \times H$ such that the symmetric bicharacter $\left.B B^{\mathrm{op}}\right|_{(K \cap H) \times(K \cap H)}$ is nondegenerate.

Proof. (i) Note that $\mathcal{S}(K, H, B)$ is nondegenerate if and only if $\mathcal{Z}_{2}(\mathcal{S}(K, H, B))=\mathcal{S}(\{e\}, G, 1)$, by [M1, Corollary 2.16]. By Proposition 4.4, this is the case if and only if $\operatorname{ker} \varphi_{B,\left(B^{\mathrm{op}}\right)^{-1}}=\{e\}$, $H K=G$, and $\psi_{B,\left(B^{\mathrm{op}}\right)^{-1}}=1$. The condition $\operatorname{ker} \varphi_{B,\left(B^{\mathrm{op}}\right)^{-1}}=\{e\}$ is equivalent to the condition that $\left.\left.B(a, \cdot)\right|_{H \cap K} B^{\mathrm{op}}(a, \cdot)\right|_{H \cap K}$ is nontrivial on $H \cap K$ whenever $a \in H \cap K-\{e\}$, that is, $\left.B B^{\mathrm{op}}\right|_{(K \cap H) \times(K \cap H)}$ is nondegenerate. The condition $\psi_{B,\left(B^{\mathrm{op}}\right)^{-1}}=1$ is automatically true when $\operatorname{ker} \varphi_{B,\left(B^{\text {op }}\right)^{-1}}=\{e\}$.

(ii) This follows immediately from Theorem 3.12, Remark 3.8 (ii), and part (i).

Remark 4.6. Let $G:=\mathbb{Z} / p^{n} \mathbb{Z}$, where $p$ is a prime. It was shown in [M1] that for $p=2$ the category $\operatorname{Rep}(D(G))$ is prime and that for odd $p$ nontrivial proper modular subcategories of $\operatorname{Rep}(D(G))$ are in bijection with isomorphisms $G \stackrel{\sim}{\rightarrow} \widehat{G}$, where $\widehat{G}$ denotes the set of group homomorphisms from $G$ to $k^{\times}$. Let us recover this result from Proposition 4.5. Suppose that $\mathcal{S}(K, H, B)$ where $(G,\{e\}) \neq(K, H) \neq(\{e\}, G)$ is a nondegenerate subcategory of $\operatorname{Rep}(D(G))$. The condition $H K=G$ implies that at least one of the subgroups $H, K$ coincides with $G$. Let us assume $H=G$ and suppose that $|K|=m<p^{n}$. Let $x, y \in G$ be generators of $H$ and $K$ respectively. Then $B(x, y)$ is an $m$ th root of 1 , so $B(y, y)$ is a root of 1 of order less than $m$ and $\left.B B^{o p}\right|_{K \times K}=\left.B^{2}\right|_{K \times K}$ is degenerate. Thus we must have $H=K=G$ and $B$ must be a (necessarily symmetric) bicharacter on $G$ such that $B^{2}$ is nondegenerate. This is impossible for $p=2$. When $p$ is odd $B^{2}$ is nondegenerate if and only if $B$ is nondegenerate, i.e., when $B$ comes from an isomorphism $G \stackrel{\sim}{\rightarrow} \widehat{G}$.

As was observed by Müger in [M1], prime nondegenerate categories serve as building blocks for nondegenerate categories. Precisely, it was shown in [M1, Theorem 4.5] that every nondegenerate category is equivalent to a finite direct product of prime ones (note, however, that such a decomposition is not unique in general). Below we collect some remarks concerning the primality of $\operatorname{Rep}(D(G))$.

Remark 4.7. (i) If $G=M \times N$ is a direct product of non-trivial groups $M$ and $N$, then $\operatorname{Rep}(D(G))$ is not prime. Indeed, by Proposition 4.5, the (nontrivial proper) subcategory $\mathcal{S}(M \times\{e\},\{e\} \times N, 1) \subseteq \operatorname{Rep}(D(G))$ is nondegenerate. This fact was also observed by Müger in [M1]. 
(ii) If $G$ is simple and nonabelian, then it immediately follows from Proposition 4.5 that $\operatorname{Rep}(D(G))$ is prime. Note that the aforementioned statement is not true for abelian simple groups [M1] (see also Remark 4.6).

(iii) As mentioned above, simplicity of a nonabelian group $G$ is sufficient for $\operatorname{Rep}(D(G))$ to be prime, however, it is not necessary. Indeed, take $G=S_{n}, n \geq 3$, and let $K, H$ be a pair of centralizing normal subgroups of $G$ such that $H K=G$. Note that $K \cap H$, being a central subgroup of $G$, must be trivial, as the center of $G$ is trivial. This implies that $G$ is a direct product of $H$ and $K$. However, as is well known, $S_{n}$ does not admit a non-trivial direct product decomposition, therefore, $\operatorname{Rep}\left(D\left(S_{n}\right)\right)$ is prime by Proposition 4.5.

4.2. The Gauss sum and central charge. Let $\mathcal{D}$ be a premodular category with twist $\theta$. Recall that the Gauss sum of $\mathcal{D}$ is defined by

$$
\tau(\mathcal{D})=\sum_{X \in \operatorname{Irr}(\mathcal{D})} \theta(X) d(X)^{2}
$$

where $\operatorname{Irr}(\mathcal{D})$ is the set of isomorphism classes of simple objects in $\mathcal{D}$. The central charge is defined by

$$
\zeta(\mathcal{D})=\frac{\tau(\mathcal{D})}{\sqrt{\operatorname{dim}(\mathcal{D})}} .
$$

For basic properties of Gauss sum and central charge we refer the reader to [BK, Sect. 3.1]. In particular, the central charge of a non-degenerate braided fusion category is known to be a root of unity (this statement is known as Vafa's theorem, see [BK, Thm. 3.1.19]).

Proposition 4.8. The Gauss sum of the fusion subcategory $\mathcal{S}(K, H, B) \subseteq \operatorname{Rep}(D(G))$ is

$$
\tau(\mathcal{S}(K, H, B))=\frac{|G|}{|H|} \sum_{a \in K \cap H \cap R}\left|K_{a}\right| B(a, a) .
$$

When $\mathcal{S}(K, H, B)$ is nondegenerate its Gauss sum is

$$
\tau(\mathcal{S}(K, H, B))=\frac{|K|}{|K \cap H|} \sum_{a \in K \cap H} B(a, a) .
$$

and its central charge is

$$
\zeta(\mathcal{S}(K, H, B))=\frac{1}{\sqrt{|K \cap H|}} \sum_{a \in K \cap H} B(a, a) .
$$

Proof. We have

$$
\begin{aligned}
\tau(\mathcal{S}(K, H, B)) & =\sum_{\text {simple }(a, \chi) \in \mathcal{S}(K, H, B)} \theta(a, \chi) d(a, \chi)^{2} \\
& =\sum_{\text {simple }} \sum_{(a, \chi) \in \mathcal{S}(K, H, B)} \frac{\chi(a)}{\operatorname{deg} \chi}\left|K_{a}\right|^{2}(\operatorname{deg} \chi)^{2}
\end{aligned}
$$

By definition of $\mathcal{S}(K, H, B)$ the above expression is equal to

$$
\sum_{a \in K \cap R}\left(\left|K_{a}\right|^{2} \sum_{\chi \in \operatorname{Irr}\left(C_{G}(a)\right):\left.\chi\right|_{H}=B(a, \cdot) \operatorname{deg} \chi}(\operatorname{deg} \chi) \chi(a)\right) .
$$


Observing that $B(a, \cdot)$ is $C_{G}(a)$-invariant and applying Frobenius reciprocity and Clifford theory, the above expression is equal to

$$
\sum_{a \in K \cap R}\left(\left|K_{a}\right|^{2} \sum_{\chi \text { is an irr. constituent of } \operatorname{Ind}_{H}^{C_{G}^{(a)}} B(a, \cdot)}(\operatorname{deg} \chi) \chi(a)\right) .
$$

Applying Frobenius reciprocity again, the above expression is equal to

$$
\sum_{a \in K \cap R}\left(\left|K_{a}\right|^{2}\left(\operatorname{Ind}_{H}^{C_{G}(a)} B(a, \cdot)\right)(a)\right) .
$$

Finally, using the formula for the induced character and the fact that the induced character is zero on $C_{G}(a) \backslash H$ the above expression is equal to

$$
\begin{aligned}
& \sum_{a \in K \cap H \cap R}\left(\frac{\left|K_{a}\right|^{2}}{|H|} \sum_{x \in C_{G}(a)} B\left(a, x^{-1} a x\right)\right) \\
= & \sum_{a \in K \cap H \cap R} \frac{\left|K_{a}\right|^{2}\left|C_{G}(a)\right|}{|H|} B(a, a) \\
= & \frac{|G|}{|H|} \sum_{a \in K \cap H \cap R}\left|K_{a}\right| B(a, a) .
\end{aligned}
$$

When $\mathcal{S}(K, H, B)$ is nondegenerate, by Proposition 4.5, $H K=G$, so $K \cap H$ is contained in the center of $G$. Therefore, the Gauss sum is

$$
\begin{aligned}
\tau(\mathcal{S}(K, H, B)) & =\frac{|G|}{|H|} \sum_{a \in K \cap H \cap R}\left|K_{a}\right| B(a, a) \\
& =\frac{|K|}{|K \cap H|} \sum_{a \in K \cap H} B(a, a)
\end{aligned}
$$

and the central charge is

$$
\begin{aligned}
\zeta(\mathcal{S}(K, H, B)) & =\frac{\tau(\mathcal{S}(K, H, B))}{\sqrt{\operatorname{dim}(\mathcal{S}(K, H, B))}} \\
& =\frac{\frac{|K|}{|K \cap H|} \sum_{a \in K \cap H} B(a, a)}{\sqrt{|K| \cdot[G: H]}} \\
& =\frac{1}{\sqrt{|K \cap H|}} \sum_{a \in K \cap H} B(a, a) .
\end{aligned}
$$

Remark 4.9. Note that the sum $\sum_{a \in K \cap H} B(a, a)$ is the classical Gauss sum for the quadratic form $a \mapsto B(a, a)$ on the Abelian group $K \cap H$. 
4.3. The central series. Following Bruguières $\left[\mathrm{Br}\right.$, we say that a functor $F: \mathcal{C}_{1} \rightarrow \mathcal{C}_{2}$ between semisimple categories $\mathcal{C}_{1}$ and $\mathcal{C}_{2}$ is dominant if every simple object in $\mathcal{C}_{2}$ is a direct summand of the image (under $F$ ) of some object in $\mathcal{C}_{1}$.

Lemma 4.10. The fusion subcategory $\mathcal{S}(K, H, 1) \subseteq \operatorname{Rep}(D(G))$ is generated by the set $\operatorname{gens}(\mathcal{S}(K, H, 1)):=\{(a, 1) \in \Gamma \mid a \in K \cap R\} \cup\left\{(e, \chi) \in \Gamma|\chi|_{H}=\operatorname{deg} \chi\right\}$.

Proof. The lemma follows immediately from the observation that the restriction functors $\operatorname{Rep}(G / H) \rightarrow \operatorname{Rep}\left(C_{G}(a) / H\right), a \in K \cap R$, are dominant.

Proposition 4.11. The adjoint subcategory $\mathcal{S}(K, H, 1)_{\text {ad }}$ of the fusion subcategory $\mathcal{S}(K, H, 1)$ of $\operatorname{Rep}(D(G))$ is $\mathcal{S}\left([G, K], C_{G}(K) \cap \pi^{-1}(Z(G / H)), 1\right)$, where $\pi$ is the canonical surjection from $G$ to $G / H$ and $[G, K]=\left\langle g \mathrm{~kg}^{-1} \mathrm{k}^{-1} \mid g \in G, k \in K\right\rangle$.

Proof. By Müger's double centralizer theorem and Lemma 3.11 it suffices to show that $\left(\mathcal{S}(K, H, 1)_{a d}\right)^{\prime}=\mathcal{S}\left(C_{G}(K) \cap \pi^{-1}(Z(G / H)),[G, K], 1\right)$. By [GN, Proposition 6.7], the simple objects (up to isomorphism) of $\left(\mathcal{S}(K, H, 1)_{a d}\right)^{\prime}$ are given by the set

$$
\begin{aligned}
\operatorname{Irr} & \left(\left(\mathcal{S}(K, H, 1)_{a d}\right)^{\prime}\right) \\
= & \left\{\left(b, \chi^{\prime}\right) \in \Gamma\left|S\left((a, \chi),\left(b, \chi^{\prime}\right)\right)\right|=d(a, \chi) d\left(b, \chi^{\prime}\right), \text { for all simple }(a, \chi) \in \mathcal{S}(K, H, 1)\right\} \\
= & \left\{\left(b, \chi^{\prime}\right) \in \Gamma|| S\left((a, \chi),\left(b, \chi^{\prime}\right)\right) \mid=d(a, \chi) d\left(b, \chi^{\prime}\right), \text { for all }(a, \chi) \in \operatorname{gens}(\mathcal{S}(K, H, 1))\right\} \\
= & \left\{\left(b, \chi^{\prime}\right) \in \Gamma|\chi|_{[G, b]}=\operatorname{deg} \chi, \text { for all } \chi \in \operatorname{Irr}(\operatorname{Rep}(G / H)),\left[K_{a}, K_{b}\right]=\{e\},\right. \\
& \text { and } \left.\left.\chi^{\prime}\right|_{[G, a]}=\operatorname{deg} \chi^{\prime}, \text { for all } a \in K \cap R\right\} \\
= & \left\{\left(b, \chi^{\prime}\right) \in \Gamma \mid b \in C_{G}(K),[G, b] \in H \text { and }\left.\chi^{\prime}\right|_{[G, K]}=\operatorname{deg} \chi^{\prime}\right\} \\
= & \left\{\left(b, \chi^{\prime}\right) \in \Gamma \mid b \in C_{G}(K) \cap \pi^{-1}(Z(G / H)), \text { and }\left.\chi^{\prime}\right|_{[G, K]}=\operatorname{deg} \chi^{\prime}\right\} \\
= & \operatorname{Irr}\left(\mathcal{S}\left(C_{G}(K) \cap \pi^{-1}(Z(G / H)),[G, K], 1\right)\right) .
\end{aligned}
$$

In the second equality above we used Lemma 4.10 and in the third equality we used Note 3.5.

Corollary 4.12. (i) The nth term of the upper central series of $\operatorname{Rep}(D(G))$ is given by

$$
\operatorname{Rep}(D(G))^{(n)}=\mathcal{S}\left(C_{n}(G), C_{G}\left(C_{n-1}(G)\right) \cap C^{n}(G), 1\right), \quad n \geq 1 .
$$

(ii) The nth term of the lower central series of $\operatorname{Rep}(D(G))$ is given by

$$
\operatorname{Rep}(D(G))_{(n)}=\mathcal{S}\left(C_{G}\left(C_{n-1}(G)\right) \cap C^{n}(G), C_{n}(G), 1\right), \quad n \geq 1 .
$$

Here $C_{n}(G)$ and $C^{n}(G)$ are the $n$th terms of the lower and upper central series of $G$, respectively.

Proof. Part (i) follows immediately from Proposition 4.11, while part (ii) follows from [GN, Theorem 6.8] and Lemma 3.11.

\section{Fusion Subcategories of the twisted Quantum Double of a Finite group}

5.1. The twisted quantum double of a finite group. Recall that a normalized 3-cocycle $\omega: G \times G \times G \rightarrow k^{\times}$is a map satisfying:

$$
\begin{gathered}
\omega\left(g_{2}, g_{3}, g_{4}\right) \omega\left(g_{1}, g_{2} g_{3}, g_{4}\right) \omega\left(g_{1}, g_{2}, g_{3}\right)=\omega\left(g_{1} g_{2}, g_{3}, g_{4}\right) \omega\left(g_{1}, g_{2}, g_{3} g_{4}\right), \\
\omega(g, e, l)=1,
\end{gathered}
$$


for all $g, l, g_{1}, g_{2}, g_{3}, g_{4} \in G$. It follows that $\omega(e, g, l)=1=\omega(g, l, e)$ for all $g, l \in G$ as well. We may assume the values of $\omega$ are roots of unity. Define

$$
\begin{aligned}
& \beta_{a}(x, y)=\frac{\omega(a, x, y) \omega\left(x, y, y^{-1} x^{-1} a x y\right)}{\omega\left(x, x^{-1} a x, y\right)} \\
& \eta_{a}(x, y)=\frac{\omega(x, y, a) \omega\left(x y a y^{-1} x^{-1}, x, y\right)}{\omega\left(x, y a y^{-1}, y\right)} \\
& \gamma_{a}(x, y)=\frac{\omega(x, y, a) \omega\left(a, a^{-1} x a, a^{-1} y a\right)}{\omega\left(x, a, a^{-1} y a\right)} \\
& \nu_{a}(x, y)=\frac{\omega\left(a x a^{-1}, a y a^{-1}, a\right) \omega(a, x, y)}{\omega\left(a x a^{-1}, a, y\right)}
\end{aligned}
$$

for all $a, x, y \in G$. Since $\omega$ is a 3-cocycle, we have

$$
\beta_{a}(x, y) \beta_{a}(x y, z)=\beta_{a}(x, y z) \beta_{x^{-1} a x}(y, z)
$$

for all $a, x, y, z \in G$. Therefore, for any $a \in G,\left.\beta_{a}\right|_{C_{G}(a)}$ is a 2-cocycle. Note that

$$
\left.\beta_{a}\right|_{C_{G}(a)}=\left.\eta_{a}\right|_{C_{G}(a)}=\left.\gamma_{a}\right|_{C_{G}(a)}=\left.\nu_{a}\right|_{C_{G}(a)} \text {. }
$$

Direct calculations, using (12), show that the following identities hold:

$$
\begin{aligned}
\frac{\gamma_{a b}(x, y)}{\gamma_{b}\left(a^{-1} x a, a^{-1} y a\right) \gamma_{a}(x, y)} & =\frac{\beta_{x}(a, b) \beta_{y}(a, b)}{\beta_{x y}(a, b)} \\
\frac{\nu_{a b}(x, y)}{\nu_{a}\left(b x b^{-1}, b y b^{-1}\right) \nu_{b}(x, y)} & =\frac{\eta_{x}(a, b) \eta_{y}(a, b)}{\eta_{x y}(a, b)}
\end{aligned}
$$

for all $a, b, x, y \in G$.

Let $\mathcal{C}=\operatorname{Rep}\left(D^{\omega}(G)\right)$. It is well known that $\mathcal{C}$ is a modular category $\mathrm{AC2}$. The set of isomorphism classes of simple objects in $\mathcal{C}$ may be identified with the set

$$
\Gamma:=\left\{(a, \chi) \mid a \in R \text { and } \chi \text { is an irreducible } \beta_{a} \text {-character of } C_{G}(a)\right\},
$$

where a $\beta_{a}$-character of $C_{G}(a)$ is the trace function of a $k^{\beta_{a}}\left[C_{G}(a)\right]$-module.

The following lemma is proved in [NN, Lemma 4.2].

Lemma 5.1. Two objects $(a, \chi),\left(b, \chi^{\prime}\right) \in \Gamma$ centralize each other if and only if the following conditions hold:

(i) The conjugacy classes $K_{a}, K_{b}$ commute element-wise.

(ii) For all $x, y \in G$,

$\left(\frac{\beta_{a}\left(x, y^{-1} b y\right) \beta_{a}\left(x y^{-1} b y, x^{-1}\right) \beta_{b}\left(y, x^{-1} a x\right) \beta_{b}\left(y x^{-1} a x, y^{-1}\right)}{\beta_{a}\left(x, x^{-1}\right) \beta_{b}\left(y, y^{-1}\right)}\right) \chi\left(x y^{-1} b y x^{-1}\right) \chi^{\prime}\left(y x^{-1} a x y^{-1}\right)=\operatorname{deg} \chi \operatorname{deg} \chi^{\prime}$.

The lemma has the following useful consequence: Taking the magnitude on both sides of the equation in Lemma 5.1(ii), we obtain

$$
\left|\chi\left(x y^{-1} b y x^{-1}\right) \chi^{\prime}\left(y x^{-1} a x y^{-1}\right)\right|=\operatorname{deg} \chi \operatorname{deg} \chi^{\prime},
$$

since the values of $\beta_{a}$ and $\beta_{b}$ are roots of unity. Now, for any $h \in C_{G}(a), \chi(h)$ is a sum of $\operatorname{deg} \chi$ roots of unity, and so by the triangle inequality, $|\chi(h)| \leq \operatorname{deg} \chi$, with equality if and only if all roots are the same. Similarly for $\chi^{\prime}$. Thus if $(a, \chi),\left(b, \chi^{\prime}\right)$ centralize each other, then $\frac{\chi\left(x y^{-1} b y x^{-1}\right)}{\operatorname{deg} \chi}$ and $\frac{\chi^{\prime}\left(y x^{-1} a x y^{-1}\right)}{\operatorname{deg} \chi^{\prime}}$ are roots of unity for all $x, y \in G$. 
5.2. Canonical data determined by a fusion subcategory of $\operatorname{Rep}\left(D^{\omega}(G)\right)$. Let $\mathcal{D}$ be a fusion subcategory of $\mathcal{C}$. Define two normal subgroups of $G$ as follows:

$$
\begin{aligned}
& K_{\mathcal{D}}=\left\{g a g^{-1} \mid g \in G,(a, \chi) \in \mathcal{D} \cap \Gamma \text { for some } \chi\right\} \\
& H_{\mathcal{D}}=\bigcap_{\chi:(e, \chi) \in \mathcal{D}} \operatorname{Ker}(\chi)
\end{aligned}
$$

where $\operatorname{Ker}(\chi)=\{g \in G \mid \chi(g)=\chi(e)\}$. Note that the definition of $H_{\mathcal{D}}$ is equivalent to $\mathcal{D} \cap \operatorname{Rep}(G)=\operatorname{Rep}\left(G / H_{\mathcal{D}}\right)$ as before.

Proposition 5.2. Let $\mathcal{D}$ be a fusion subcategory of $\mathcal{C}$. Then $H_{\mathcal{D}^{\prime}}=K_{\mathcal{D}}, K_{\mathcal{D}^{\prime}}=H_{\mathcal{D}}$, and the groups $K_{\mathcal{D}}$ and $H_{\mathcal{D}}$ centralize each other.

Proof. By Lemma 5.1 a simple object $(e, \chi)$ in $\operatorname{Rep}(G)$ centralizes $\mathcal{D}$ if and only if $\chi(a)=$ $\operatorname{deg} \chi$ for all $a \in K_{\mathcal{D}}$. The rest is similar to the proof of Proposition 3.6.

Lemma 5.3. Let $\mathcal{E}$ be a fusion subcategory of $\mathcal{C}$ and let $(a, \chi) \in \mathcal{E} \cap \Gamma$. Suppose $\chi^{\prime}$ is an irreducible $\beta_{a}$-character of $C_{G}(a)$ such that $\frac{\left.\chi^{\prime}\right|_{K_{\mathcal{E}^{\prime}}}}{\operatorname{deg} \chi^{\prime}}=\frac{\left.\chi\right|_{K_{\mathcal{E}^{\prime}}}}{\operatorname{deg} \chi}$. Then $\left(a, \chi^{\prime}\right) \in \mathcal{E}$.

Proof. We will show that $\left(a, \chi^{\prime}\right)$ centralizes $\mathcal{E}^{\prime}$. Let $\left(b, \chi^{\prime \prime}\right) \in \mathcal{E}^{\prime} \cap \Gamma$. Note that since $(a, \chi) \in \mathcal{E}$, the conjugacy classes $K_{a}$ and $K_{b}$ commute. It remains to show that the equation in Lemma 5.1(ii) holds for the pair $\left(a, \chi^{\prime}\right),\left(b, \chi^{\prime \prime}\right)$. By hypothesis, $\frac{\left.\chi^{\prime}\right|_{\mathcal{E}_{\mathcal{E}}^{\prime}}}{\operatorname{deg} \chi^{\prime}}=\frac{\left.\chi\right|_{K_{\mathcal{E}^{\prime}}}}{\operatorname{deg} \chi}$, so we may replace $\chi^{\prime}$ by $\chi$ in the equation. However we already know that $(a, \chi)$ centralizes $\mathcal{E}^{\prime}$ since $(a, \chi) \in \mathcal{E}$, so by Lemma 5.1(ii), the desired equation holds.

Let $\mathcal{D}$ be any fusion subcategory of $\mathcal{C}$. Define a pairing

$$
B_{\mathcal{D}}: K_{\mathcal{D}} \times H_{\mathcal{D}} \rightarrow k^{\times}
$$

as follows. Let $(a, \chi) \in \mathcal{D} \cap \Gamma$, and

$$
B_{\mathcal{D}}\left(x^{-1} a x, h\right):=\frac{\beta_{a}(x, h) \beta_{a}\left(x h, x^{-1}\right)}{\beta_{a}\left(x, x^{-1}\right)} \frac{\chi\left(x h x^{-1}\right)}{\operatorname{deg} \chi}
$$

for all $x \in G, h \in H_{\mathcal{D}}$. We will show that $B_{\mathcal{D}}$ is well-defined and does not depend on $\chi$. For this, we give an equivalent definition: Write $h=y^{-1} b y$ for $b \in H_{\mathcal{D}} \cap R, y \in G$, $\left(b, \chi^{\prime}\right) \in \mathcal{D}^{\prime} \cap \Gamma$. Since $(a, \chi)$ and $\left(b, \chi^{\prime}\right)$ centralize each other, we have by the definition (22) of $B_{\mathcal{D}}$ and Lemma 5.1(ii) that

$$
B_{\mathcal{D}}\left(x^{-1} a x, y^{-1} b y\right)=\frac{\beta_{b}\left(y, y^{-1}\right)}{\beta_{b}\left(y, x^{-1} a x\right) \beta_{b}\left(y x^{-1} a x, y^{-1}\right)} \frac{\operatorname{deg} \chi^{\prime}}{\chi^{\prime}\left(y x^{-1} a x y^{-1}\right)} .
$$

This proves that $B_{\mathcal{D}}$ does not depend on the choice of $\chi$. Now suppose $z^{-1} a z=x^{-1} a x$ for some $z \in G$. Then by (23),

$$
\begin{aligned}
B_{\mathcal{D}}\left(z^{-1} a z, y^{-1} b y\right) & =\frac{\beta_{b}\left(y, y^{-1}\right)}{\beta_{b}\left(y, z^{-1} a z\right) \beta_{b}\left(y z^{-1} a z, y^{-1}\right)} \frac{\operatorname{deg} \chi^{\prime}}{\chi^{\prime}\left(y z^{-1} a z y^{-1}\right)} \\
& =\frac{\beta_{b}\left(y, y^{-1}\right)}{\beta_{b}\left(y, x^{-1} a x\right) \beta_{b}\left(y x^{-1} a x, y^{-1}\right)} \frac{\operatorname{deg} \chi^{\prime}}{\chi^{\prime}\left(y x^{-1} a x y^{-1}\right)} \\
& =B_{\mathcal{D}}\left(x^{-1} a x, y^{-1} b y\right) .
\end{aligned}
$$

Therefore $B_{\mathcal{D}}$ is well-defined. 
Next we give a definition of a $G$-invariant $\omega$-bicharacter, generalizing [NN, Defns. 4.5, 4.6] (the case $K=H$ ), and show that $B_{\mathcal{D}}$ satisfies the definition.

Definition 5.4. Let $H, K$ be normal subgroups of $G$ that centralize each other, and let $B: K \times H \rightarrow k^{\times}$be a function.

We say that $B$ is an $\omega$-bicharacter if

$$
\begin{aligned}
\text { (i) } B(x, y z) & =\beta_{x}^{-1}(y, z) B(x, y) B(x, z) \text { and } \\
\text { (ii) } B(w x, y) & =\beta_{y}(w, x) B(w, y) B(x, y)
\end{aligned}
$$

for all $w, x \in K, y, z \in H$. Equivalently, $B(x, \cdot)$ is a $\beta_{x}$-character of $H$ and $B(-, y)$ is a $\beta_{y}^{-1}$-character of $K$.

We say that $B$ is $G$-invariant if

$$
B\left(x^{-1} k x, h\right)=\frac{\beta_{k}(x, h) \beta_{k}\left(x h, x^{-1}\right)}{\beta_{k}\left(x, x^{-1}\right)} B\left(k, x h x^{-1}\right)
$$

for all $x, y \in G, h \in H, k \in K$.

Remark 5.5. (i) The $G$-invariance property corresponds to the following action of $G$ : Let $\left\{\delta_{x}\right\}_{x \in G}$ be the basis of the linear dual $(k G)^{*}$ of the group algebra, that is dual to $G$, that is $\delta_{x}(y)=\delta_{x, y}$ for all $x, y \in G$. Then we may identify a basis of $D^{\omega}(G)$ with the set $\left\{\delta_{x} \bar{g}\right\}_{x, g \in G}$. In this notation, the subalgebra $k \delta_{x} C_{G}(x)$ of $D^{\omega}(G)$ is isomorphic to the twisted group algebra $k^{\beta_{x}}\left[C_{G}(x)\right]$. A group element $g \in G$ acts on $D^{\omega}(G)$ via conjugation by the invertible element $\bar{g}=\sum_{x \in G} \delta_{x} \bar{g}$, and takes the subalgebra $k^{\beta_{x}}\left[C_{G}(x)\right]$ to $k^{\beta_{g^{-1} x g}}\left[C_{G}\left(g^{-1} x g\right)\right]$. Under these identifications, $B$ is $G$-invariant if and only if the function $B(k, \cdot)$ on $k^{\beta_{k}}[H]$ is taken to the function $B\left(x^{-1} k x, \cdot\right)$ on $k^{\beta_{x-1} k x}[H]$ via conjugation by $\bar{x}$.

(ii) There is a symmetry in the definition of an $\omega$-bicharacter that appears to be lacking in the definition of $G$-invariance. However, a consequence of the next lemma is that there is an equivalent definition of $G$-invariance, considering $B$ instead to be a function in the first argument, fixing the second.

Lemma 5.6. Let $x, h, k \in G$ for which $h k=k h$. Then

(i) $\frac{\nu_{x}(h, k)}{\nu_{x}(k, h)}=\frac{\beta_{x h x^{-1}}\left(x, x^{-1}\right)}{\beta_{x h x^{-1}}(x, k) \beta_{x h x^{-1}}\left(x k, x^{-1}\right)}$,

(ii) $\frac{\nu_{x}\left(x^{-1} h x, x^{-1} k x\right)}{\nu_{x}\left(x^{-1} k x, x^{-1} h x\right)}=\frac{\nu_{x^{-1}}(k, h)}{\nu_{x^{-1}}(h, k)}$, and

(iii) $\frac{\beta_{k}\left(y^{-1}, y\right)}{\beta_{k}\left(y^{-1}, h\right) \beta_{k}\left(y^{-1} h, y\right)}=\frac{\beta_{h}\left(y, y^{-1}\right)}{\beta_{h}(y, k) \beta_{h}\left(y k, y^{-1}\right)}$.

Proof. (i) We must show that

$$
\frac{\nu_{x}(h, k)}{\nu_{x}(k, h)} \cdot \frac{\beta_{x h x^{-1}}(x, k) \beta_{x h x^{-1}}\left(x k, x^{-1}\right)}{\beta_{x h x^{-1}}\left(x, x^{-1}\right)}=1 .
$$

This follows by applying the definitions (17) and (14), and the 3-cocycle condition (12) to the tuples $\left(x k x^{-1}, x, h, x^{-1}\right),\left(x k x^{-1}, x h x^{-1}, x, x^{-1}\right),\left(x h x^{-1}, x k x^{-1}, x, x^{-1}\right),\left(x k x^{-1}, x, x^{-1}, x h x^{-1}\right)$. 
(ii) Applying (21) to the quadruples $\left(x, x^{-1}, h, k\right)$ and $\left(x, x^{-1}, k, h\right)$ we obtain:

$$
\begin{aligned}
\nu_{x}\left(x^{-1} h x, x^{-1} k x\right) \nu_{x^{-1}}(h, k) & =\frac{\eta_{h k}\left(x, x^{-1}\right)}{\eta_{h}\left(x, x^{-1}\right) \eta_{k}\left(x, x^{-1}\right)}, \\
\nu_{x}\left(x^{-1} k x, x^{-1} h x\right) \nu_{x^{-1}}(k, h) & =\frac{\eta_{k h}\left(x, x^{-1}\right)}{\eta_{k}\left(x, x^{-1}\right) \eta_{h}\left(x, x^{-1}\right)} .
\end{aligned}
$$

Since $h k=k h$ the stated identity follows.

(iii) Applying the identity in part (i) to the triples $\left(y^{-1}, y k y^{-1}, h\right)$ and $\left(y, y^{-1} h y, k\right)$, the claimed identity is equivalent to

$$
\frac{\nu_{y^{-1}}\left(y k y^{-1}, h\right) \nu_{y}\left(k, y^{-1} h y\right)}{\nu_{y^{-1}}\left(h, y k y^{-1}\right) \nu_{y}\left(y^{-1} h y, k\right)}=1
$$

which is seen to be true by applying the identity in part (ii) to the triple $\left(y, y k y^{-1}, h\right)$.

It follows from part (iii) of the lemma that a function $B: K \times H \rightarrow k^{\times}$is $G$-invariant if and only if

$$
B\left(k, y^{-1} h y\right)=\frac{\beta_{h}\left(y, y^{-1}\right)}{\beta_{h}(y, k) \beta_{h}\left(y k, y^{-1}\right)} B\left(y k y^{-1}, h\right)
$$

for all $x, y \in G, h \in H, k \in K$.

Proposition 5.7. Let $\mathcal{D}$ be a fusion subcategory of $\mathcal{C}$. Then $B_{\mathcal{D}}$ is a $G$-invariant $\omega$ bicharacter on $K_{\mathcal{D}} \times H_{\mathcal{D}}$.

Proof. First we check $G$-invariance. By definition (22), for all $a \in K \cap R$, we have

$$
B_{\mathcal{D}}\left(x^{-1} a x, h\right)=\frac{\beta_{a}(x, h) \beta_{a}\left(x h, x^{-1}\right)}{\beta_{a}\left(x, x^{-1}\right)} \frac{\chi\left(x h x^{-1}\right)}{\operatorname{deg} \chi}=\frac{\beta_{a}(x, h) \beta_{a}\left(x h, x^{-1}\right)}{\beta_{a}\left(x, x^{-1}\right)} B_{\mathcal{D}}\left(a, x h x^{-1}\right),
$$

the second equality by (22) with $h$ replaced by $x h x^{-1}$ and $x$ replaced by the identity element. More generally, let $k=y^{-1} a y$, and apply the above equation twice, the first time to express $B\left((y x)^{-1} a y x, h\right)$ as a scalar multiple of $B\left(a,(y x) h(y x)^{-1}\right)$, and the second time to express $B\left(a, y\left(x h x^{-1}\right) y^{-1}\right)$ as a scalar multiple of $B\left(y^{-1} a y, x h x^{-1}\right)$. We obtain in this way:

$$
B\left(x^{-1} k x, h\right)=\frac{\beta_{a}\left(y, y^{-1}\right) \beta_{a}(y x, h) \beta_{a}\left(y x h, x^{-1} y^{-1}\right)}{\beta_{a}\left(y, x h x^{-1}\right) \beta_{a}\left(y x h x^{-1}, y^{-1}\right) \beta_{a}\left(y x, x^{-1} y^{-1}\right)} B\left(k, x h x^{-1}\right) .
$$

This is equivalent to $G$-invariance: Apply (18) thrice to

$$
\frac{\beta_{y^{-1} a y}(x, h) \beta_{y^{-1} a y}\left(x h, x^{-1}\right)}{\beta_{y^{-1} a y}\left(x, x^{-1}\right)}
$$

to express all scalars as images of $\beta_{a}$, and then apply (18) to the quadruples $\left(a, y x h, x^{-1}, y^{-1}\right)$ and $\left(a, y x, x^{-1}, y^{-1}\right)$ to obtain the expression (24).

Now let $h_{1}, h_{2} \in H$ and $a \in K \cap R$. By the definition of $B_{\mathcal{D}}$, we have

$$
B_{\mathcal{D}}\left(a, h_{1}\right) B_{\mathcal{D}}\left(a, h_{2}\right)=\frac{\chi\left(h_{1}\right)}{\operatorname{deg} \chi} \frac{\chi\left(h_{2}\right)}{\operatorname{deg} \chi}=\beta_{a}\left(h_{1}, h_{2}\right) \frac{\chi\left(h_{1} h_{2}\right)}{\operatorname{deg} \chi}=\beta_{a}\left(h_{1}, h_{2}\right) B_{\mathcal{D}}\left(a, h_{1} h_{2}\right) .
$$


Let $x \in G$. By $G$-invariance of $B_{\mathcal{D}}$ we now have that $B_{\mathcal{D}}\left(x^{-1} a x, h_{1}\right) B_{\mathcal{D}}\left(x^{-1} a x, h_{2}\right)$ is equal to

$$
\begin{aligned}
& \frac{\beta_{a}\left(x, h_{1}\right) \beta_{a}\left(x h_{1}, x^{-1}\right) \beta_{a}\left(x, h_{2}\right) \beta_{a}\left(x h_{2}, x^{-1}\right)}{\beta_{a}\left(x, x^{-1}\right)^{2}} B\left(a, x h_{1} x^{-1}\right) B\left(a, x h_{2} x^{-1}\right) \\
& =\frac{\beta_{a}\left(x h_{1} x^{-1}, x h_{2} x^{-1}\right) \beta_{a}\left(x, h_{1}\right) \beta_{a}\left(x h_{1}, x^{-1}\right) \beta_{a}\left(x, h_{2}\right) \beta_{a}\left(x h_{2}, x^{-1}\right)}{\beta_{a}\left(x, x^{-1}\right)^{2}} B\left(a, x h_{2} h_{2} x^{-1}\right) .
\end{aligned}
$$

On the other hand,

$$
\beta_{x^{-1} a x}\left(h_{1}, h_{2}\right) B_{\mathcal{D}}\left(x^{-1} a x, h_{1} h_{2}\right)=\frac{\beta_{x^{-1} a x}\left(h_{1}, h_{2}\right) \beta_{a}\left(x, h_{1} h_{2}\right) \beta_{a}\left(x h_{1} h_{2}, x^{-1}\right)}{\beta_{a}\left(x, x^{-1}\right)} B\left(a, x h_{1} h_{2} x^{-1}\right) .
$$

Comparing, the two will be the same if and only if

$$
\frac{\beta_{a}\left(x h_{1} x^{-1}, x h_{2} x^{-1}\right) \beta_{a}\left(x, h_{1}\right) \beta_{a}\left(x h_{1}, x^{-1}\right) \beta_{a}\left(x, h_{2}\right) \beta_{a}\left(x h_{2}, x^{-1}\right)}{\beta_{a}\left(x, x^{-1}\right) \beta_{x^{-1} a x}\left(h_{1}, h_{2}\right) \beta_{a}\left(x, h_{1} h_{2}\right) \beta_{a}\left(x h_{1} h_{2}, x^{-1}\right)}=1 .
$$

This we prove just as in the proof of [NN, Lemma 4.10]: We apply (18) for $\beta_{a}$ successively to the five triples $\left(x, h_{1}, h_{2}\right),\left(x, h_{2}, x^{-1}\right),\left(x h_{1}, x^{-1}, x h_{2} x^{-1}\right),\left(x h_{1}, h_{2}, x^{-1}\right),\left(x, x^{-1}, x h_{2} x^{-1}\right)$ and make a corresponding substitution each time. It follows that $B_{\mathcal{D}}$ satisfies the first equation in the definition of $\omega$-bicharacter. To see that it satisfies the second, we use the alternative definition (23) of $B_{\mathcal{D}}$, and apply an analogous argument.

We have associated, to each fusion subcategory $\mathcal{D}$ of $\mathcal{C}$, a canonical triple $\left(K_{\mathcal{D}}, H_{\mathcal{D}}, B_{\mathcal{D}}\right)$, where $K_{\mathcal{D}}$ and $H_{\mathcal{D}}$ are normal subgroups of $G$ centralizing each other, and $B_{\mathcal{D}}$ is a $G$-invariant $\omega$-bicharacter. Next we associate to each triple a fusion subcategory.

5.3. Construction of a fusion subcategory of $\operatorname{Rep}\left(D^{\omega}(G)\right)$. Let $K, H$ be normal subgroups of $G$ that centralize each other, and $B: K \times H \rightarrow k^{\times}$a $G$-invariant $\omega$-bicharacter. Define

$\mathcal{S}(K, H, B):=$ full Abelian subcategory of $\mathcal{C}$ generated by

$$
\left\{\begin{array}{l|l}
(a, \chi) \in \Gamma \mid \begin{array}{l}
a \in K \cap R \text { and } \chi \text { is an irreducible } \beta_{a} \text {-character of } C_{G}(a) \\
\text { such that } \chi(h)=B(a, h) \operatorname{deg} \chi, \text { for all } h \in H
\end{array}
\end{array}\right\} .
$$

We will prove that $\mathcal{S}(K, H, B)$ is a fusion subcategory of $\mathcal{C}$ and determine its centralizer. We will need [NN, Lemma 4.4], proved by Clifford theory [Ka, Theorem 7.8.1] and Frobenius reciprocity [Ka, Proposition 7.4.8] for projective characters:

Lemma 5.8. Let $E$ be a normal subgroup of a finite group $F$, and $\alpha$ a 2-cocycle on $F$. Let $\operatorname{Irr}(F)$ denote the set of irreducible $\alpha$-projective characters of $F$. Let $\rho$ be an $F$-invariant

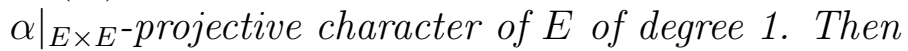

$$
\sum_{\chi \in \operatorname{Irr}(F):\left.\chi\right|_{E}=(\operatorname{deg} \chi) \rho}(\operatorname{deg} \chi)^{2}=[F: E] .
$$

To apply the lemma to our situation, replace $E$ by $H$ and $F$ by $C_{G}(a)$, and note that by $G$-invariance of $B$, the function $B(a,-)$ is a $C_{G}(a)$-invariant $\left.\beta_{a}\right|_{H \times H \text {-projective character }}$ of $H$ of degree 1. One consequence of the lemma then is that there exists at least one $\chi \in \operatorname{Irr}\left(C_{G}(a)\right)$ such that $(a, \chi) \in \mathcal{S}(K, H, B)$. This implies in particular that $K$ is the support of $\mathcal{S}(K, H, B)$. 
Lemma 5.9. The dimension of $\mathcal{S}(K, H, B)$ is $|K|[G: H]$.

Proof. The proof is the same as for $\omega=1$ (Lemma 3.10), replacing Lemma 3.9 by Lemma 5.8,

Let $\left(B^{\mathrm{op}}\right)^{-1}: H \times K \rightarrow k^{\times}$be the function defined by $\left(B^{\mathrm{op}}\right)^{-1}(h, k)=B(k, h)^{-1}$. Then $\left(B^{\text {op }}\right)^{-1}$ is a $G$-invariant $\omega$-bicharacter, by symmetry of Definition 5.4 (see Remark 5.5(ii)).

Lemma 5.10. $\mathcal{S}(K, H, B)$ is a fusion subcategory of $\mathcal{C}$ and $\mathcal{S}(K, H, B)^{\prime}=\mathcal{S}\left(H, K,\left(B^{\mathrm{op}}\right)^{-1}\right)$.

Proof. First we show that $\mathcal{S}(K, H, B)$ and $\mathcal{S}\left(H, K,\left(B^{\mathrm{op}}\right)^{-1}\right)$ centralize each other. Let $(a, \chi) \in \mathcal{S}(K, H, B) \cap \Gamma$ and $\left(b, \chi^{\prime}\right) \in \mathcal{S}\left(H, K,\left(B^{\text {op }}\right)^{-1}\right) \cap \Gamma$. Then the conjugacy classes $K_{a}$ and $K_{b}$ centralize each other. We check Lemma 5.1(ii), using the two equations (22) and (23):

$$
\begin{aligned}
& \frac{\beta_{a}\left(x, y^{-1} b y\right) \beta_{a}\left(x y^{-1} b y, x^{-1}\right) \beta_{b}\left(y, x^{-1} a x\right) \beta_{b}\left(y x^{-1} a x, y^{-1}\right)}{\beta_{a}\left(x, x^{-1}\right) \beta_{b}\left(y, y^{-1}\right)} \chi\left(x y^{-1} b y x^{-1}\right) \chi^{\prime}\left(y x^{-1} a x y^{-1}\right) \\
& =\frac{\beta_{a}\left(x, y^{-1} b y\right) \beta_{a}\left(x y^{-1} b y, x^{-1}\right) \beta_{b}\left(y, x^{-1} a x\right) \beta_{b}\left(y x^{-1} a x, y^{-1}\right)}{\beta_{a}\left(x, x^{-1}\right) \beta_{b}\left(y, y^{-1}\right)} \\
& \cdot B\left(a, x y^{-1} b y x^{-1}\right) \operatorname{deg} \chi\left(B^{\mathrm{op}}\right)^{-1}\left(b, y x^{-1} a x y^{-1}\right) \operatorname{deg} \chi^{\prime} .
\end{aligned}
$$

By $G$-invariance, this may be rewritten as

$$
B\left(x^{-1} a x, y^{-1} b y\right) \operatorname{deg} \chi B^{-1}\left(x^{-1} a x, y^{-1} b y\right) \operatorname{deg} \chi^{\prime}=\operatorname{deg} \chi \operatorname{deg} \chi^{\prime},
$$

as desired. Therefore $\mathcal{S}(K, H, B)$ and $\mathcal{S}\left(H, K,\left(B^{\mathrm{op}}\right)^{-1}\right)$ centralize each other.

By Lemma 5.9.

$$
\operatorname{dim}(\mathcal{S}(K, H, B)) \operatorname{dim}\left(\mathcal{S}\left(H, K,\left(B^{\text {op }}\right)^{-1}\right)=|G|^{2}=\operatorname{dim} \mathcal{C} .\right.
$$

By Lemma 2.1, $\mathcal{S}(K, H, B)$ is a fusion subcategory of $\mathcal{C}$ and $\mathcal{S}(K, H, B)^{\prime}=\mathcal{S}\left(H, K,\left(B^{\text {op }}\right)^{-1}\right)$.

Theorem 5.11. The assignments $\mathcal{D} \mapsto\left(K_{\mathcal{D}}, H_{\mathcal{D}}, B_{\mathcal{D}}\right)$ and $(K, H, B) \mapsto \mathcal{S}(K, H, B)$ are inverses of each other. Thus there is a bijection between the set of fusion subcategories of $\mathcal{C}$ and triples $(K, H, B)$ where $K, H$ are normal subgroups of $G$ that centralize each other and $B: K \times H \mapsto k^{\times}$is a $G$-invariant $\omega$-bicharacter.

Proof. First we show that $\left(K_{\mathcal{S}(K, H, B)}, H_{\mathcal{S}(K, H, B)}, B_{\mathcal{S}(K, H, B)}\right)=(K, H, B)$. We have already

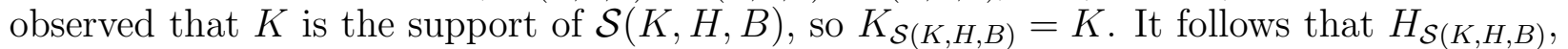
which is the support of $\mathcal{S}(K, H, B)^{\prime}=\mathcal{S}\left(H, K,\left(B^{\mathrm{op}}\right)^{-1}\right)$, is $H$. Let $a \in K \cap R,(a, \chi) \in$ $\mathcal{S}(K, H, B) \cap \Gamma$, and $h \in H$. Then by the definition of $B_{\mathcal{S}(K, H, B)}$, we have

$$
B_{\mathcal{S}(K, H, B)}(a, h)=\frac{\chi(h)}{\operatorname{deg} \chi} .
$$

On the other hand, since $(a, \chi) \in \mathcal{S}(K, H, B)$, we have $\chi(h)=B(a, h) \operatorname{deg} \chi$, so $B(a, h)=$ $B_{\mathcal{S}(K, H, B)}(a, h)$. By $G$-invariance, this implies $B=B_{\mathcal{S}(K, H, B)}$.

Now we show that $\mathcal{S}\left(K_{\mathcal{D}}, H_{\mathcal{D}}, B_{\mathcal{D}}\right)=\mathcal{D}$. Let $(a, \chi) \in \mathcal{S}\left(K_{\mathcal{D}}, H_{\mathcal{D}}, B_{\mathcal{D}}\right) \cap \Gamma$. Then for all $h \in H_{\mathcal{D}}$, by the definitions of $\mathcal{S}\left(K_{\mathcal{D}}, H_{\mathcal{D}}, B_{\mathcal{D}}\right)$ and of $B_{\mathcal{D}}$, respectively, we have

$$
\frac{\chi(h)}{\operatorname{deg} \chi}=B_{\mathcal{D}}(a, h)=\frac{\chi^{\prime}(h)}{\operatorname{deg} \chi^{\prime}}
$$


for any $\chi^{\prime}$ such that $\left(a, \chi^{\prime}\right)$ is simple in $\mathcal{D}$. By Proposition 5.2 , we have $K_{\mathcal{D}^{\prime}}=H_{\mathcal{D}}$, so the above equation holds for all $h \in K_{\mathcal{D}^{\prime}}$. By Lemma [5.3, we have $(a, \chi) \in \mathcal{D}$. We have shown $\mathcal{S}\left(K_{\mathcal{D}}, H_{\mathcal{D}}, B_{\mathcal{D}}\right) \subseteq \mathcal{D}$. Similarly $\mathcal{S}\left(K_{\mathcal{D}^{\prime}}, H_{\mathcal{D}^{\prime}}, B_{\mathcal{D}^{\prime}}\right)=\mathcal{S}\left(H_{\mathcal{D}}, K_{\mathcal{D}}, B_{\mathcal{D}^{\prime}}\right) \subseteq \mathcal{D}^{\prime}$. By Lemma 5.9 ,

$$
\operatorname{dim}\left(\mathcal{S}\left(K_{\mathcal{D}}, H_{\mathcal{D}}, B_{\mathcal{D}}\right)\right) \operatorname{dim}\left(\mathcal{S}\left(H_{\mathcal{D}}, K_{\mathcal{D}}, B_{\mathcal{D}^{\prime}}\right)\right)=|G|^{2}=\operatorname{dim} \mathcal{C} .
$$

This forces $\mathcal{S}\left(H_{\mathcal{D}}, K_{\mathcal{D}}, B_{\mathcal{D}^{\prime}}\right)=\mathcal{S}\left(K_{\mathcal{D}}, H_{\mathcal{D}}, B_{\mathcal{D}}\right)^{\prime}$, so that $\mathcal{D}^{\prime} \subseteq \mathcal{S}\left(H_{\mathcal{D}}, K_{\mathcal{D}}, B_{\mathcal{D}^{\prime}}\right)$. Therefore $\mathcal{D}^{\prime}=\mathcal{S}\left(H_{\mathcal{D}}, K_{\mathcal{D}}, B_{\mathcal{D}^{\prime}}\right)$, and by symmetry, $\mathcal{D}=\mathcal{S}\left(K_{\mathcal{D}}, H_{\mathcal{D}}, B_{\mathcal{D}}\right)$.

Remark 5.12. Let $\mathcal{C}$ be any braided group-theoretical fusion category (see Section 2.1). The braiding yields a canonical embedding of $\mathcal{C}$ into its center $\mathcal{Z}(\mathcal{C})$. (See $[\mathrm{K}]$ for the center construction.) Since $\mathcal{C}$ is group-theoretical, $\mathcal{Z}(\mathcal{C})$ is equivalent to $\mathcal{Z}\left(\operatorname{Vec}_{G}^{\omega}\right) \cong \operatorname{Rep}\left(D^{\omega}(G)\right)$ for some finite group $G$ and 3-cocycle $\omega$. (This follows from [ENO, Sect. 8.8]. See also the proof of [Na, Thm. 1.2] and [Mj, Prop. 3.3].) Therefore $\mathcal{C}$ may be realized as a fusion subcategory of $\operatorname{Rep}\left(D^{\omega}(G)\right)$. By Theorem 5.11, $\mathcal{C}$ is equivalent to a category of the form $\mathcal{S}(K, H, B)$ defined in (25) for some normal subgroups $K, H$ of $G$ that centralize each other and $G$-invariant $\omega$-bicharacter $B$ on $K \times H$.

\section{Some invariants and the lattice of Fusion subcategories of $\operatorname{Rep}\left(D^{\omega}(G)\right)$}

6.1. The lattice. Let $K, H, K^{\prime}, H^{\prime}$ be normal subgroups of $G$ such that $K$ and $H$ centralize each other, and $K^{\prime}$ and $H^{\prime}$ centralize each other. Let $B: K \times H \rightarrow k^{\times}$and $B^{\prime}: K^{\prime} \times H^{\prime} \rightarrow k^{\times}$ be $G$-invariant $\omega$-bicharacters.

Proposition 6.1. $\mathcal{S}(K, H, B) \subseteq \mathcal{S}\left(K^{\prime}, H^{\prime}, B^{\prime}\right)$ if and only if $K \subseteq K^{\prime}, H^{\prime} \subseteq H$, and $\left.B\right|_{K \times H^{\prime}}=\left.B^{\prime}\right|_{K \times H^{\prime}}$.

Proof. The statement follows from the definitions since $K$ is the support of $S(K, H, B)$ by Lemma 5.8 .

Let $\varphi_{B, B^{\prime}}: K \cap K^{\prime} \rightarrow \widehat{H \cap H^{\prime}}$ be the function defined by

$$
\varphi_{B, B^{\prime}}(a)=\left.\left.B(a, \cdot)\right|_{H \cap H^{\prime}}\left(B^{\prime}\right)^{-1}(a, \cdot)\right|_{H \cap H^{\prime}}
$$

where $\widehat{H \cap H^{\prime}}$ denotes the group of group homomorphisms from $H \cap H^{\prime}$ to $k^{\times}$. It is straightforward to check that for each $a \in K \cap K^{\prime}, \varphi_{B, B^{\prime}}(a)$ is indeed a group homomorphism from $H \cap H^{\prime}$ to $k^{\times}$, and that $\varphi_{B, B^{\prime}}$ is itself a group homomorphism from $K \cap K^{\prime}$ to $\widehat{H \cap H^{\prime}}$. Let $\psi_{B, B^{\prime}}: \operatorname{ker} \varphi_{B, B^{\prime}} \times H H^{\prime} \rightarrow k^{\times}$be the function defined by

$$
\psi_{B, B^{\prime}}\left(a, h h^{\prime}\right)=\beta_{a}^{-1}\left(h, h^{\prime}\right) B(a, h) B^{\prime}\left(a, h^{\prime}\right)
$$

for all $a \in K, h \in H, h^{\prime} \in H^{\prime}$. Note that $\psi_{B, B^{\prime}}$ is well-defined: Suppose $h_{1} h_{1}^{\prime}=h_{2} h_{2}^{\prime}$ for elements $h_{1}, h_{2} \in H, h_{1}^{\prime}, h_{2}^{\prime} \in H^{\prime}$. We must check that

$$
\beta_{a}^{-1}\left(h_{1}, h_{1}^{\prime}\right) B\left(a, h_{1}\right) B^{\prime}\left(a, h_{1}^{\prime}\right)=\beta_{a}^{-1}\left(h_{2}, h_{2}^{\prime}\right) B\left(a, h_{2}\right) B^{\prime}\left(a, h_{2}^{\prime}\right),
$$

or equivalently,

$$
\beta_{a}^{-1}\left(h_{1}, h_{1}^{\prime}\right) B\left(a, h_{2}\right)^{-1} B\left(a, h_{1}\right)=\beta_{a}^{-1}\left(h_{2}, h_{2}^{\prime}\right) B^{\prime}\left(a, h_{2}^{\prime}\right) B^{\prime}\left(a, h_{1}^{\prime}\right)^{-1} .
$$

Since $B, B^{\prime}$ are $\omega$-bicharacters, we have

$$
B\left(a, h_{2}\right) B\left(a, h_{2}^{-1}\right)=\beta_{a}\left(h_{2}, h_{2}^{-1}\right) B(a, 1)=\beta_{a}\left(h_{2}, h_{2}^{-1}\right),
$$


so $B\left(a, h_{2}\right)^{-1}=\beta_{a}^{-1}\left(h_{2}, h_{2}^{-1}\right) B\left(a, h_{2}^{-1}\right)$, and similarly, $B^{\prime}\left(a, h_{1}^{\prime}\right)^{-1}=\beta_{a}^{-1}\left(h_{1}^{\prime},\left(h_{1}^{\prime}\right)^{-1}\right) B\left(a,\left(h_{1}^{\prime}\right)^{-1}\right)$. Thus we must show that

$$
\frac{\beta_{a}\left(h_{2}^{-1}, h_{1}\right)}{\beta_{a}\left(h_{1}, h_{1}^{\prime}\right) \beta_{a}\left(h_{2}, h_{2}^{-1}\right)} B\left(a, h_{2}^{-1} h_{1}\right)=\frac{\beta_{a}\left(h_{2}^{\prime},\left(h_{1}^{\prime}\right)^{-1}\right)}{\beta_{a}\left(h_{2}, h_{2}^{\prime}\right) \beta_{a}\left(h_{1}^{\prime},\left(h_{1}^{\prime}\right)^{-1}\right)} B^{\prime}\left(a, h_{2}^{\prime}\left(h_{1}^{\prime}\right)^{-1}\right) .
$$

Now, $h_{2}^{-1} h_{1}=h_{2}^{\prime}\left(h_{1}^{\prime}\right)^{-1} \in H \cap H^{\prime}$, and since $a \in \operatorname{ker} \varphi_{B, B^{\prime}}$, we have $B\left(a, h_{2}^{-1} h_{1}\right)=$ $B^{\prime}\left(a, h_{2}^{\prime}\left(h_{1}^{\prime}\right)^{-1}\right)$. Thus we must show that

$$
1=\frac{\beta_{a}\left(h_{1}, h_{1}^{\prime}\right) \beta_{a}\left(h_{2}, h_{2}^{-1}\right) \beta_{a}\left(h_{2}^{\prime},\left(h_{1}^{\prime}\right)^{-1}\right)}{\beta_{a}\left(h_{2}^{-1}, h_{1}\right) \beta_{a}\left(h_{2}, h_{2}^{\prime}\right) \beta_{a}\left(h_{1}^{\prime},\left(h_{1}^{\prime}\right)^{-1}\right)} .
$$

This follows by applying the 2-cocycle identity for $\beta_{a}$ successively to the triples $\left(h_{2}^{-1}, h_{1}, h_{1}^{\prime}\right)$, $\left(h_{2}^{-1}, h_{2}, h_{2}^{\prime}\right)$, and $\left(h_{2}^{\prime},\left(h_{1}^{\prime}\right)^{-1}, h_{1}^{\prime}\right)$. Therefore $\psi_{B, B^{\prime}}$ is well-defined.

Lemma 6.2. The function $\psi_{B, B^{\prime}}$ is a $G$-invariant $\omega$-bicharacter on $\operatorname{ker} \varphi_{B, B^{\prime}} \times H H^{\prime}$.

Proof. We first prove that identity (ii) in Definition 5.4 holds for $\psi_{B, B^{\prime}}$ : Let $a, a^{\prime} \in \operatorname{ker} \varphi_{B, B^{\prime}}$, and $h \in H, h^{\prime} \in H^{\prime}$. We must prove

$$
\psi_{B, B^{\prime}}\left(a a^{\prime}, h h^{\prime}\right)=\beta_{h h^{\prime}}\left(a, a^{\prime}\right) \psi_{B, B^{\prime}}\left(a, h h^{\prime}\right) \psi_{B, B^{\prime}}\left(a^{\prime}, h h^{\prime}\right) .
$$

The left side is

$$
\frac{1}{\beta_{a a^{\prime}}\left(h, h^{\prime}\right)} B\left(a a^{\prime}, h\right) B^{\prime}\left(a a^{\prime}, h^{\prime}\right)=\frac{\beta_{h}\left(a, a^{\prime}\right) \beta_{h^{\prime}}\left(a, a^{\prime}\right)}{\beta_{a a^{\prime}}\left(h, h^{\prime}\right)} B(a, h) B\left(a^{\prime}, h\right) B^{\prime}\left(a, h^{\prime}\right) B^{\prime}\left(a^{\prime}, h^{\prime}\right) .
$$

The right side is

$$
\frac{\beta_{h h^{\prime}}\left(a, a^{\prime}\right)}{\beta_{a}\left(h, h^{\prime}\right) \beta_{a^{\prime}}\left(h, h^{\prime}\right)} B(a, h) B^{\prime}\left(a, h^{\prime}\right) B\left(a^{\prime}, h\right) B^{\prime}\left(a^{\prime}, h^{\prime}\right) .
$$

The two are equal if and only if

$$
1=\frac{\beta_{h}\left(a, a^{\prime}\right) \beta_{h^{\prime}}\left(a, a^{\prime}\right) \beta_{a}\left(h, h^{\prime}\right) \beta_{a^{\prime}}\left(h, h^{\prime}\right)}{\beta_{a a^{\prime}}\left(h, h^{\prime}\right) \beta_{h h^{\prime}}\left(a, a^{\prime}\right)} .
$$

This is true by the identity (20) and by (19) (note $a, a^{\prime}$ centralize $h, h^{\prime}$ ).

Next we prove that $\psi_{B, B^{\prime}}$ is $G$-invariant: We must prove that for all $x \in G$,

$$
\psi_{B, B^{\prime}}\left(x^{-1} a x, h h^{\prime}\right)=\frac{\beta_{a}\left(x, h h^{\prime}\right) \beta_{a}\left(x h h^{\prime}, x^{-1}\right)}{\beta_{a}\left(x, x^{-1}\right)} \psi_{B, B^{\prime}}\left(a, x h h^{\prime} x^{-1}\right) .
$$

By the definition of $\psi_{B, B^{\prime}}$ and $G$-invariance of $B, B^{\prime}$, the left side is

$$
\begin{aligned}
& \frac{1}{\beta_{x^{-1} a x}\left(h, h^{\prime}\right)} B\left(x^{-1} a x, h\right) B^{\prime}\left(x^{-1} a x, h^{\prime}\right) \\
& =\frac{\beta_{a}(x, h) \beta_{a}\left(x h, x^{-1}\right) \beta_{a}\left(x, h^{\prime}\right) \beta_{a}\left(x h^{\prime}, x^{-1}\right)}{\beta_{x^{-1} a x}\left(h, h^{\prime}\right) \beta_{a}\left(x, x^{-1}\right)^{2}} B\left(a, x h x^{-1}\right) B^{\prime}\left(a, x h^{\prime} x^{-1}\right) .
\end{aligned}
$$

The right side is

$$
\frac{\beta_{a}\left(x, h h^{\prime}\right) \beta_{a}\left(x h h^{\prime}, x^{-1}\right)}{\beta_{a}\left(x, x^{-1}\right) \beta_{a}\left(x h x^{-1}, x h^{\prime} x^{-1}\right)} B\left(a, x h x^{-1}\right) B^{\prime}\left(a, x h^{\prime} x^{-1}\right) .
$$

So the left side equals the right side if and only if

$$
1=\frac{\beta_{a}(x, h) \beta_{a}\left(x h, x^{-1}\right) \beta_{a}\left(x, h^{\prime}\right) \beta_{a}\left(x h^{\prime}, x^{-1}\right) \beta_{a}\left(x h x^{-1}, x h^{\prime} x^{-1}\right)}{\beta_{x^{-1} a x}\left(h, h^{\prime}\right) \beta_{a}\left(x, x^{-1}\right) \beta_{a}\left(x, h h^{\prime}\right) \beta_{a}\left(x h h^{\prime}, x^{-1}\right)} .
$$


This follows by applying the identity (18) to the tuples $\left(a, x, h, h^{\prime}\right),\left(a, x h, x^{-1}, x h^{\prime} x^{-1}\right)$, $\left(a, x, x^{-1}, x h^{\prime} x^{-1}\right),\left(a, x h, h^{\prime}, x^{-1}\right)$, and $\left(a, x, h^{\prime}, x^{-1}\right)$.

Finally we prove that identity (i) in Definition 5.4 holds for $\psi_{B, B^{\prime}}$. Let $a \in \operatorname{ker} \varphi_{B, B^{\prime}}$ and $y_{1}, z_{1} \in H, y_{2}, z_{2} \in H^{\prime}, y=y_{1} y_{2}, z=z_{1} z_{2}$. We show that

$$
\psi_{B, B^{\prime}}(a, y z)=\beta_{a}^{-1}(y, z) \psi_{B, B^{\prime}}(a, y) \psi_{B, B^{\prime}}(a, z) .
$$

Since $B^{\prime}$ is $G$-invariant and $z_{1} \in C_{G}(a)$, the left side $\psi_{B, B^{\prime}}\left(a,\left(y_{1} z_{1}\right)\left(z_{1}^{-1} y_{2} z_{1} z_{2}\right)\right)$ is equal to

$$
\begin{aligned}
&= \beta_{a}^{-1}\left(y_{1} z_{1}, z_{1}^{-1} y_{2} z_{1} z_{2}\right) B\left(a, y_{1} z_{1}\right) B^{\prime}\left(a, z_{1}^{-1} y_{2} z_{1} z_{2}\right) \\
&= \beta_{a}^{-1}\left(y_{1} z_{1}, z_{1}^{-1} y_{2} z_{1} z_{2}\right) \beta_{a}^{-1}\left(y_{1}, z_{1}\right) \beta_{a}^{-1}\left(z_{1}^{-1} y_{2} z_{1}, z_{2}\right) B\left(a, y_{1}\right) B\left(a, z_{1}\right) B^{\prime}\left(a, z_{1}^{-1} y_{2} z_{1}\right) B^{\prime}\left(a, z_{2}\right) \\
&= \frac{\beta_{a}\left(z_{1}^{-1}, z_{1}\right)}{\beta_{a}\left(y_{1} z_{1}, z_{1}^{-1} y_{2} z_{1} z_{2}\right) \beta_{a}\left(y_{1}, z_{1}\right) \beta_{a}\left(z_{1}^{-1} y_{2} z_{1}, z_{2}\right) \beta_{a}\left(z_{1}^{-1}, y_{2}\right) \beta_{a}\left(z_{1}^{-1} y_{2}, z_{1}\right)} \\
& \cdot B\left(a, y_{1}\right) B\left(a, z_{1}\right) B^{\prime}\left(a, y_{2}\right) B^{\prime}\left(a, z_{2}\right) .
\end{aligned}
$$

The right side is

$$
\beta_{a}^{-1}\left(y_{1} y_{2}, z_{1} z_{2}\right) \beta_{a}^{-1}\left(y_{1}, y_{2}\right) \beta_{a}^{-1}\left(z_{1}, z_{2}\right) B\left(a, y_{1}\right) B^{\prime}\left(a, y_{2}\right) B\left(a, z_{1}\right)^{\prime}\left(a, z_{2}\right) .
$$

The two are equal if and only if

$$
1=\frac{\beta_{a}\left(z_{1}^{-1}, z_{1}\right) \beta_{a}\left(y_{1}, y_{2}\right) \beta_{a}\left(z_{1}, z_{2}\right) \beta_{a}\left(y_{1} y_{2}, z_{1} z_{2}\right)}{\beta_{a}\left(y_{1} z_{1}, z_{1}^{-1} y_{2} z_{1} z_{2}\right) \beta_{a}\left(y_{1}, z_{1}\right) \beta_{a}\left(z_{1}^{-1} y_{2} z_{1}, z_{2}\right) \beta_{a}\left(z_{1}^{-1}, y_{2}\right) \beta_{a}\left(z_{1}^{-1} y_{2}, z_{1}\right)} .
$$

This follows from the identity $\beta_{a}\left(z_{1}^{-1}, z_{1}\right)=\beta_{a}\left(z_{1}, z_{1}^{-1}\right)$ (obtained by applying the 2-cocycle identity to $\left.\left(z_{1}^{-1}, z_{1}, z_{1}^{-1}\right)\right)$ and application of the 2-cocycle identity for $\beta_{a}$ to the triples $\left(y_{1}, z_{1}, z_{1}^{-1}\right),\left(z_{1}^{-1}, y_{2}, z_{1}\right),\left(z_{1}^{-1}, y_{2} z_{1}, z_{2}\right),\left(y_{2}, z_{1}, z_{2}\right),\left(y_{1} z_{1}, z_{1}^{-1} y_{2}, z_{1} z_{2}\right)$, and $\left(y_{1} z_{1}, z_{1}^{-1}, y_{2}\right)$.

Proposition 6.3. $\quad$ (i) $\mathcal{S}(K, H, B) \cap \mathcal{S}\left(K^{\prime}, H^{\prime}, B^{\prime}\right)=\mathcal{S}\left(\operatorname{ker} \varphi_{B, B^{\prime}}, H H^{\prime}, \psi_{B, B^{\prime}}\right)$.

(ii) $\left.\mathcal{S}(K, H, B) \vee \mathcal{S}\left(K^{\prime}, H^{\prime}, B^{\prime}\right)=\mathcal{S}\left(K K^{\prime}, \operatorname{ker} \varphi_{B^{\mathrm{op}},\left(B^{\prime}\right){ }^{\mathrm{op}}},\left(\psi_{B^{\mathrm{op}},\left(B^{\prime}\right)^{\mathrm{op}}}\right)^{\mathrm{op}}\right)\right)$.

Proof. The proof is the same as for $\omega=1$ (Proposition 4.2), using Lemma 5.10 in place of Lemma 3.11 .

We will need the following lemma in order to characterize isotropic subcategories.

Lemma 6.4. Let $B: K \times H \rightarrow k^{\times}$be a G-invariant $\omega$-bicharacter with $K \subseteq H$ and $B(a, a)=1$, for all $a \in K \cap R$. Then $B(k, k)=1$, for all $k \in K$.

Proof. Let $k \in K$ and write $k=x^{-1} a x, x \in G, a \in K \cap R$. By $G$-invariance of $B$ and the equality $B(a, a)=1$ we obtain

$$
B\left(x^{-1} a x, x^{-1} a x\right)=\frac{\beta_{a}\left(x, x^{-1} a x\right) \beta_{a}\left(a x, x^{-1}\right)}{\beta_{a}\left(x, x^{-1}\right)} .
$$

Applying the definition (14) of $\beta_{a}$ and the 3-cocycle condition (12) for $\omega$ to the quadruples $\left(a, x, x^{-1} a x, x^{-1}\right)$ and $\left(a, x, x^{-1}, a\right)$, the right side of the above equality is equal to 1 .

We next characterize symmetric, isotropic and Lagrangian subcategories. Definitions were given in Section 4.1 .

Proposition 6.5. The fusion subcategory $\mathcal{S}(K, H, B) \subseteq \operatorname{Rep}\left(D^{\omega}(G)\right)$ is

(i) symmetric if and only if $K \subseteq H$ and $B\left(k_{1}, k_{2}\right) B\left(k_{2}, k_{1}\right)=1$, for all $k_{1}, k_{2} \in K$,

(ii) isotropic if and only if $K \subseteq H$ and $\left.B\right|_{K \times K}$ is alternating, 
(iii) Lagrangian if and only $K=H$ and $B$ is alternating.

Proof. The proof is the same as for $\omega=1$ (Proposition 4.3), using Lemma 5.10 and Proposition 6.1 in place of Lemma 3.11 and Proposition 4.1. We note that in part (ii) we also use Lemma 6.4.

Proposition 6.6. $\mathcal{Z}_{2}(\mathcal{S}(K, H, B))=\mathcal{S}\left(\operatorname{ker} \varphi_{B,\left(B^{\mathrm{op}}\right)^{-1}}, H K, \psi_{B,\left(B^{\mathrm{op}}\right)^{-1}}\right)$.

Proof. The proof is the same as for $\omega=1$ (Proposition 4.4), using Lemma 5.10 and Proposition 6.3(i) in place of Lemma 3.11 and Proposition 4.2(i).

Note that if $B$ is an $\omega$-bicharacter on $K \times H$, then $B^{\text {op }}$ is an $\omega^{-1}$-bicharacter on $H \times K$. Consequently, $B B^{\mathrm{op}}$ is a (symmetric) bicharacter on $(K \cap H) \times(K \cap H)$.

Proposition 6.7. $\quad$ (i) The fusion subcategory $\mathcal{S}(K, H, B) \subseteq \operatorname{Rep}\left(D^{\omega}(G)\right)$ is nondegenerate if and only if $H K=G$ and the symmetric bicharacter $\left.B B^{\mathrm{op}}\right|_{(K \cap H) \times(K \cap H)}$ is nondegenerate.

(ii) $\operatorname{Rep}\left(D^{\omega}(G)\right)$ is prime if and only if there is no triple $(K, H, B)$, where $K$ and $H$ are normal subgroups of $G$ that centralize each other, $(G,\{e\}) \neq(K, H) \neq(\{e\}, G)$, $H K=G$, and $B$ is a $G$-invariant $\omega$-bicharacter on $K \times H$ such that $\left.B B^{\mathrm{op}}\right|_{(K \cap H) \times(K \cap H)}$ is nondegenerate.

Proof. The proof is the same as for $\omega=1$ (Proposition 4.5), using Theorem 5.11 and Proposition 6.6 in place of Theorem 3.12 and Proposition 4.4.

6.2. The Gauss sum and central charge. The definitions of Gauss sum and central charge of a premodular category were recalled in Section 4.2.

Proposition 6.8. The Gauss sum of the fusion subcategory $\mathcal{S}(K, H, B)$ of $\operatorname{Rep}\left(D^{\omega}(G)\right)$ is

$$
\tau(\mathcal{S}(K, H, B))=\frac{|G|}{|H|} \sum_{a \in K \cap H \cap R}\left|K_{a}\right| B(a, a) .
$$

When $\mathcal{S}(K, H, B)$ is nondegenerate its Gauss sum is

$$
\tau(\mathcal{S}(K, H, B))=\frac{|K|}{|K \cap H|} \sum_{a \in K \cap H} B(a, a) .
$$

and its central charge is

$$
\zeta(\mathcal{S}(K, H, B))=\frac{1}{\sqrt{|K \cap H|}} \sum_{a \in K \cap H} B(a, a) .
$$

Proof. The proof is the same as for $\omega=1$ (Proposition 4.8), using Proposition 6.7 in place of Proposition 4.5.

\section{A CHARACTERIZATION OF GROUP-THEORETICAL BRAIDED FUSION CATEGORIES}

Let us recall notions of equivariantization and de-equivariantization of fusion categories from $[\mathrm{Br}, \mathrm{M} 2, \mathrm{Ki}]$.

Let $\mathcal{C}$ be a fusion category with an action of a finite group $G$. In this case one can define the fusion category $\mathcal{C}^{G}$ of $G$-equivariant objects in $\mathcal{C}$. An object of this category is an object $X$ of $\mathcal{C}$ equipped with an isomorphism $u_{g}: g(X) \rightarrow X$ for all $g \in G$, such that

$$
u_{g h} \circ \gamma_{g, h}=u_{g} \circ g\left(u_{h}\right),
$$


where $\gamma_{g, h}: g(h(X)) \rightarrow g h(X)$ is the natural isomorphism associated to the action. Morphisms and tensor product of equivariant objects are defined in an obvious way. This category is called the $G$-equivariantization of $\mathcal{C}$.

There is a procedure opposite to equivariantization, called de-equivariantization. In the context of modular categories it was introduced as a modularization by Bruguières and Müger. Namely, let $\mathcal{C}$ be a fusion category and let $\mathcal{E}=\operatorname{Rep}(G) \subset \mathcal{Z}(\mathcal{C})$ be a Tannakian subcategory that embeds into $\mathcal{C}$ via the forgetful functor $\mathcal{Z}(\mathcal{C}) \rightarrow \mathcal{C}$. Let $A=\operatorname{Fun}(G)$ be the algebra of functions on $G$. It is a commutative algebra in $Z(\mathcal{C})$ and so the category $\mathcal{C}_{G}$ of left $A$-modules in $\mathcal{C}$ is a fusion category, called the de-equivariantization of $\mathcal{C}$ by $\mathcal{E}$. The free module functor $\mathcal{C} \rightarrow \mathcal{C}_{G}: X \mapsto A \otimes X$ is a surjective tensor functor.

The above constructions are inverse to each other. In particular, $\mathcal{C}_{G}$ admits a canonical action of $G$ such that there is a canonical equivalence $\left(\mathcal{C}_{G}\right)^{G} \cong \mathcal{C}$.

Remark 7.1. The following consequence of the above constructions will be used in the proof of Theorem 7.2 below. Given a braided fusion category $\mathcal{D}$ and a Tannakian subcategory $\operatorname{Rep}(G) \subset \mathcal{D}$ we have a commutative algebra $A=\operatorname{Fun}(G)$ in $\mathcal{D}$. The category $\mathcal{D}_{G}$ of $A$-modules in $\mathcal{D}$ is a fusion category with an action of $G$ and $\mathcal{D}$ is equivalent to the $G$ equivariantization of $\mathcal{D}_{G}$.

The next theorem characterizes group-theoretical braided fusion categories as equivariantizations of pointed fusion categories.

Theorem 7.2. Let $\mathcal{D}$ be a braided fusion category. Then $\mathcal{D}$ is group-theoretical if and only if it contains a Tannakian subcategory $\mathcal{E}=\operatorname{Rep}(G)$ such that the corresponding deequivariantization $\mathcal{D}_{G}$ is pointed. Equivalently, $\mathcal{D}$ is group-theoretical if and only if it is an equivariantization of a pointed fusion category.

Proof. It follows from [Nk, Theorem 3.5] that an equivariantization of a pointed fusion category is group-theoretical. Hence, if $\mathcal{D}_{G}$ is pointed then $\mathcal{D} \cong\left(\mathcal{D}_{G}\right)^{G}$ is group-theoretical.

Conversely, suppose $\mathcal{D}$ is group-theoretical. Then by Remark 5.12 and Theorem 5.11 , there exist a group $G$, a 3-cocycle $\omega$ on it, normal subgroups $K, H \subset G$, and $B: K \times H \rightarrow k^{\times}$such that $\mathcal{D}=\mathcal{S}(K, H, B) \subset \operatorname{Rep}\left(D^{\omega}(G)\right)$. The category $\mathcal{D}$ contains a Tannakian subcategory $\operatorname{Rep}(G / H)$ and is identified with a certain category of equivariant vector bundles on $K$. Let $\tilde{\omega}$ denote the restriction of $\omega$ to $K$, let $F: \mathcal{D} \rightarrow \operatorname{Vec}_{K}^{\tilde{\omega}}$ be the restriction of the forgetful functor $\operatorname{Rep}\left(D^{\omega}(G)\right) \cong \mathcal{Z}\left(\operatorname{Vec}_{G}^{\omega}\right) \rightarrow \operatorname{Vec}_{G}^{\omega}$, and let $I: \operatorname{Vec}_{K}^{\tilde{\omega}} \rightarrow \mathcal{D}$ be the left adjoint to $F$. Then $A:=I(\mathbf{1})=\operatorname{Fun}(G / H)$ is a commutative algebra in $\operatorname{Rep}(G / H) \subset \mathcal{D}$. Furthermore, for any $X \in \operatorname{Vec}_{K}^{\tilde{\omega}}$ the object $I(X)$ has a structure of an $A$-module (see [DGNO, proof of Theorem 4.8] for details) and the functor $X \mapsto I(X)$ is a tensor equivalence between $\operatorname{Vec}_{K}^{\tilde{\omega}}$ and the category of $A$-modules in $\mathcal{D}$. By Remark [7.1, $\mathcal{D}$ is equivalent to a $G / H$-equivariantization of the pointed category $\operatorname{Vec}_{K}^{\tilde{\omega}}$, so the proof is complete.

Remark 7.3. (i) A different proof of Theorem 7.2 will be given in an updated version of DGNO. The proof presented here uses the explicit description of group-theoretical categories as subcategories of twisted group doubles.

(ii) By the work of Kirillov Jr. [Ki] and Müger [M2] the above de-equivariantization of $\mathcal{D}$ has a structure of a braided $G$-crossed category in the sense of Turaev [T]. By definition, a braided $G$-crossed fusion category is a fusion category $\mathcal{C}$ equipped with an action $g \mapsto T_{g}$ of a group $G$ by tensor autoequivalences of $\mathcal{C}$, a (not necessarily 
faithful) grading $\mathcal{C}=\oplus_{g \in G} \mathcal{C}_{g}$, and a natural collection of isomorphisms

$$
c_{X, Y}: X \otimes Y \simeq T_{g}(Y) \otimes X, \quad X \in \mathcal{C}_{g}, g \in G \quad \text { and } \quad Y \in \mathcal{C}
$$

satisfying certain compatibility conditions.

(iii) Recall that a crossed module is a pair of groups $(G, X)$ with $G$ acting on $X$ by automorphisms, denoted $(g, x) \mapsto{ }^{g} x$, and a group homomorphism $\partial: X \rightarrow G$ satisfying

$$
{ }^{\partial(x)} x^{\prime}=x x^{\prime} x^{-1}, \quad \text { for all } x, x^{\prime} \in X
$$

and

$$
\partial\left({ }^{g} x\right)=g \partial(x) g^{-1}, \quad \text { for all } g \in G, x \in X .
$$

Let $\mathcal{C}$ be a pointed $G$-crossed braided category and let $X$ be the group of isomorphism classes of simple objects of $G$. Then $(G, X)$ is crossed module. By Theorem 7.2 every group-theoretical braided fusion category gives rise to a crossed module.

A construction of braided fusion categories from crossed modules was given by Bantay in [Ba]. But a general problem of classifying pointed $G$-crossed categories in terms of group cohomology seems to be quite complicated, see [M4, 4.9].

\section{REFERENCES}

[AC1] D. Altschuler and A. Coste, Invariants of 3-manifolds from finite groups, Proc. XXth Int. Conf. Diff. Geom. Meth. in Theor. Phys., New York, 1991, 219-233, World Scientific, 1992.

[AC2] D. Altschuler and A. Coste, Invariants of three manifolds from finite group cohomology, J. Geom. Phys. 11 (1993), 191-203.

[Ba] P. Bantay, Characters of crossed modules and premodular categories, arXiv:math/0512542v1 [math.QA].

[Br] A. Bruguières, Catégories prémodulaires, modularization et invariants des variétés de dimension 3, Mathematische Annalen, 316 (2000), no. 2, 215-236.

[BK] B. Bakalov and A. Kirillov Jr., Lectures on tensor categories and modular functors, University Lecture Series, vol. 21, AMS, RI, 2001.

[CGR] A. Coste, T. Gannon, and P. Ruelle, Finite group modular data, Nucl. Phys. B581 (2000), 679-717.

[D] P. Deligne, Catégories tannakiennes, The Grothendieck Festschrift, Vol. II, Progr. Math., 87, Birkhauser, Boston, MA, 1990, 111-195.

[DGNO] V. Drinfeld, S. Gelaki, D. Nikshych, and V. Ostrik, Group-theoretical properties of nilpotent modular categories, arXiv:0704.0195v2 [math.QA].

[DPR1] R. Dijkgraaf, V. Pasquier, and P. Roche, Quasi-quantum groups related to orbifold models, Nuclear Phys. B. Proc. Suppl. 18B (1990), 60-72.

[DPR2] R. Dijkgraaf, V. Pasquier, and P. Roche, Quasi-Hopf algebras, group cohomology, and orbifold models, Integrable systems and quantum groups (Pavia, 1990), World Sci. Publishing, River Edge, NJ, 75-98 (1992).

[ENO] P. Etingof, D. Nikshych, and V. Ostrik, On fusion categories, Ann. of Math. 162 (2005), 581-642.

[GN] S. Gelaki and D. Nikshych, Nilpotent fusion categories, Adv. Math. 217 (2008), 1053-1071.

[Ka] G. Karpilovsky, Projective representations of finite groups, Monographs and textbooks in pure and applied mathematics, Marcel Dekker, Inc, New York, 1985.

[K] C. Kassel, Quantum groups, Graduate texts in mathematics 155, Springer-Verlag, New York, 1995.

[Ki] A. Kirillov, Jr. Modular categories and orbifold models II, arXiv:math/0110221v1 [math.QA].

[Mj] S. Majid, Quantum double for quasi-Hopf algebras, Lett. Math. Phys. 45 (1998), 1-9.

[Ma] G. Mason, The quantum double of a finite group and its role in conformal field theory, London Mathematical Society Lecture Note Series 212, 405-417, Cambridge Univ. Press, Cambridge, 1995.

[M1] M. Müger, On the structure of modular categories, Proc. London Math. Soc. (3) 87 (2003), 291-308.

[M2] M. Müger, Galois extensions of braided tensor categories and braided crossed G-categories, J. Algebra 277 (2004), no. 1, 256-281. 
[M3] M. Müger, From subfactors to categories and topology I. Frobenius algebras in and Morita equivalence of tensor categories, J. Pure Appl. Algebra 180 (2003), 81-157.

[M4] M. Müger, Conformal orbifold theories and braided crossed G-categories, Comm. Math. Phys. 260 (2005),727-762.

[NN] D. Naidu and D. Nikshych, Lagrangian subcategories and braided tensor equivalences of twisted quantum doubles of finite groups, Comm. Math. Phys. 279 (2008), 845-872.

[Na] S. Natale, On group theoretical Hopf algebras and exact factorizations of finite groups, J. Algebra 270 (2003), 199-211.

[Nk] D. Nikshych, Non group-theoretical semisimple Hopf algebras from group actions on fusion categories, Selecta Math. 14 (2008), 145-161.

[O1] V. Ostrik, Module categories, weak Hopf algebras and modular invariants, Transform. Groups 8 (2003), no.2, 177-206.

[O2] V. Ostrik, Module categories over the Drinfeld double of a finite group, Int. Math. Res. Not., 2003, no. $27,1507-1520$.

[T] V. Turaev, Homotopy field theory in dimension 3 and crossed group-categories, arXiv:math/0005291.

Department of Mathematics, Texas A\&M University, College Station, TX 77843, USA

E-mail address: dnaidu@math.tamu.edu

Department of Mathematics and Statistics, University of New Hampshire, Durham, NH 03824, USA

E-mail address: nikshych@math.unh.edu

Department of Mathematics, Texas A\&M University, College Station, TX 77843, USA

E-mail address: sjw@math.tamu.edu 